\title{
LAS BARBAS DEL VECINO. LOS PATRONES DE DIFUSIÓN DEL CRIMEN VIOLENTO EN MÉXICO (1990-2010)
}

DAVID RAMÍREZ-DE-GARAY

\section{INTRODUCGIÓN: LOS ORÍGENES DEL CONTAGIO}

LA PALABRA CONTAGIO, COMO UNA ETIQUETA para identificar la difusión del crimen sobre un territorio a lo largo del tiempo, ha sido empleada en distintos ámbitos y con distintos objetivos. Uno de los más conocidos es la prevención del crimen y la violencia. Pero al hablar de prevención del crimen y de la violencia se trata de dos aproximaciones distintas hacia la prevención y al diseño de políticas públicas. La primera busca diseñar políticas públicas y estrategias de intervención (sobre todo a nivel local) para evitar la aparición de conductas delictivas o antisociales. Es una postura que surgió como una respuesta a la crisis de seguridad y justicia que se vivió en las grandes concentraciones urbanas de Occidente en la década de 1970. El alza en las tasas de crimen violento atrajo amplias críticas contra el sistema basado en la policía, los jueces y las prisiones como un método eficaz para la contención y reducción de la criminalidad. El discurso a favor de la prevención apareció como una alternativa viable y, a la larga, económica para ayudar a contener la criminalidad antes de que sucediera, por lo que rápidamente se convirtió en el cuarto pilar de las políticas de seguridad y justicia. Gran parte del trabajo teórico, empírico y de evaluación sobre este tipo de prevención fue realizado por la comunidad académica de la criminología, la sociología, la ciencia política y la psicología. 
La prevención de la violencia o el enfoque de salud pública se basa en un presupuesto distinto: la difusión de la violencia tiene características similares a las de una epidemia. Esta idea apareció a finales de la década de 1990 y rápidamente alcanzó relevancia internacional al tratar a la violencia como un problema de salud pública, valorando el costo que implicaba en los sistemas de salud alrededor del mundo. Este salto se consolidó en 1996 en la 49 Asamblea Mundial de la Salud con la resolución WHA49.25, donde se declara que la violencia es un problema de salud pública fundamental y creciente en todo el mundo. La resolución buscó llamar la atención sobre el impacto (a corto y largo plazo) que tiene la violencia más allá de la víctima y sus familiares. La principal meta de la resolución fue: "aumentar la conciencia acerca del problema de la violencia en el mundo, y dejar claro que la violencia puede prevenirse y que la salud pública tiene el cometido fundamental de abordar sus causas y consecuencias". ${ }^{1}$

A partir de esta base conceptual se continuó con un proceso de institucionalización de la prevención de la violencia. Por medio de la Organización Mundial de la Salud (oms) se formuló una definición mínima de la violencia, ${ }^{2}$ se creó una tipología de la violencia en tres categorías (violencia autoinfligida, interpersonal y colectiva) y se postuló un modelo ecológico ${ }^{3}$ amplio que ponía énfasis en los factores de riesgo, con que se logró que la estrategia

${ }^{1}$ Krug et al. (eds.), World Report on Violence and Health, Ginebra, World Health Organization, 2002, p. XxI.

2 "El uso intencional de la fuerza o el poder físico, de hecho o como amenaza, contra uno mismo, otra persona o un grupo o comunidad, que cause o tenga muchas probabilidades de causar lesiones, muerte, daños psicológicos, trastornos del desarrollo o privaciones". Krug, op. cit., p. 5.

${ }^{3} \mathrm{El}$ modelo ecológico plantea que la violencia -y los factores de riesgo asociados a ella- se puede abordar a partir de cuatro niveles interrelacionados: individual (características biológicas, impulsividad, nivel educativo, uso de drogas, agresividad, experiencia de maltrato), relacional (amistades, relaciones de pareja, contexto familiar), comunitario (contextos escolar, laboral y comunitario) y social (elementos culturales a favor de la violencia, el suicidio, dominio masculino y uso de la fuerza; así como fenómenos estructurales como la desigualdad y la salud pública). Krug, op. cit., p. 5. 
de prevención se enfocara en el control de los factores de riesgo asociados con las conductas violentas. ${ }^{4}$

El enfoque epidemiológico implica una idea particular sobre la manera en que la violencia se propaga en una zona en el transcurso del tiempo. Es la imagen de una dinámica de difusión atribuible a la presencia o no de los factores de riesgo. Este tipo de difusión es muy similar a la forma que tienen cierto tipo de enfermedades para distribuirse de manera contagiosa. Así pues, el enfoque para la prevención de la violencia implica que la violencia como fenómeno social tiene una distribución de tipo contagiosa similar a la de una enfermedad. De hecho, el símil es tan claro que se manifiesta en todo el discurso de prevención y en las políticas de implementación. ${ }^{5}$ Esta característica de la distribución de la violencia se ha convertido en una parte fundamental de todo el enfoque epidemiológico de la prevención. Sin embargo, ¿cuál es el sustento empírico de esta característica? ¿Existen trabajos que hayan abordado el tema? De ser así, ¿es una característica de todo tipo de violencia o es inherente a sólo un tipo de conducta?; ¿con qué tipo de métodos se ha analizado esta forma de dispersión?

Para responder a estas preguntas se consultaron los estudios citados por la oms y se realizó una búsqueda de trabajos académicos sobre el tema. La pesquisa arrojó que la gran mayoría de los trabajos de investigación sobre la difusión contagiosa de la violencia (o que emplean la noción de contagio como parte de su análisis) se concentran en las siguientes conductas: violencia intrafamiliar, predisposición moral a la violencia, efectos de la violencia política, suicidio y violencia de bandas. Entre los trabajos revisados, los más citados emplean distintas técnicas para detectar y evaluar la posibilidad de contagio. Las técnicas con mayor presencia son: regresión logística multivariada, ${ }^{6}$ correlaciones,

${ }^{4}$ Por medio de tres tipos de intervenciones: generales, seleccionadas e indicadas.

${ }^{5}$ Para tener una perspectiva detallada de los autores que trabajan el enfoque de prevención basado en la salud, recomiendo consultar National Research Council, Contagion of Violence: Workshop Summary, Washington, D. C., The National Academies Press, 2013.

${ }^{6}$ T. Abramsky et al., "What Factors Are Associated with Recent Intimate Part- 
ANOvA, regresión lineal, ${ }^{7}$ análisis de patrones (path analysis),${ }^{8}$ ecuaciones estructurales ${ }^{9}$ y regresiones jerárquicas. ${ }^{10}$

Los estudios relacionan el contagio de la violencia con los siguientes efectos: desorden social y generación de incivilidades, conflicto familiar, disminución de la seguridad de los niños en su entorno social a raíz de la violencia política, incremento en suicidios y mayor violencia doméstica. El vínculo entre esta serie de consecuencias y la violencia se establece al experimentar o ejercer conductas violentas en un tiempo previo. Los mecanismos que dan cuenta

ner Violence? Findings from the WHO Multi-Country Study on Women's Health and Domestic Violence”, BMC Public Health, vol. 11, núm. 1, p. 109; Hanson, SelfBrown, Fricker-Elhai, Kilpatrick, Saunders y Resnick, "Relations among Parental Substance Use, Violence Exposure and Mental Health: The National Survey of Adolescents", Addictive Behaviors, vol. 31, núm. 11, 2006, pp. 1988-2001.

${ }^{7}$ Abramsky et al., op. cit.; S. L. Berman, Kurtines, Silverman, y Serafini, "The Impact of Exposure to Crime and Violence on Urban Youth", The American Journal of Orthopsychiatry, vol. 66, núm. 3, 1996, pp. 329-336; Devries et al., "Violence against Women Is Strongly Associated with Suicide Attempts: Evidence from the WHO Multi-Country Study on Women's Health and Domestic Violence against Women”, Social Science Ẽ Medicine (1982), vol. 73, núm. 1, 2011, pp. 79-86.

${ }^{8}$ Cummings et al., "Longitudinal Pathways between Political Violence and Child Adjustment: The Role of Emotional Security about the Community in Northern Ireland", Journal of Abnormal Child Psychology, vol. 39, núm. 2, 2011, pp. 213-224; también: Cummings et al., "Political Violence and Child Adjustment in Northern Ireland: Testing Pathways in a Social-Ecological Model Including Single-and Two-Parent Families", Developmental Psychology, vol. 46, núm. 4, 2010, pp. 827-841.

${ }^{9}$ Guerra, Huesmann y Spindler, "Community Violence Exposure, Social Cognition, and Aggression among Urban Elementary School Children”, Child Development, vol. 74, núm. 5, 2003, pp. 1561-1576, en http://www.ncbi.nlm.nih.gov/ pubmed/14552414; Kokko, Pulkkinen, Huesmann, Dubow y Boxer, "Intensity of Aggression in Childhood as a Predictor of Different Forms of Adult Aggression: A Two-Country (Finland and United States) Analysis", Journal of Research on Adolescence: The Official Journal of the Society for Research on Adolescence, vol. 19, núm. 1, 2009, pp. 9-34.

${ }^{10}$ Dubow et al., "Exposure to Conflict and Violence across Contexts: Relations to Adjustment among Palestinian Children", Journal of Clinical Child and Adolescent Psychology, vol. 39, núm. 1, 2010, pp. 103-116, en doi:10.1080/15374410903401153; Ehrensaft et al., "Intergenerational Transmission of Partner Violence: A 20-Year Prospective Study", Journal of Consulting and Clinical Psychology, vol. 71, núm. 4, 2003, pp. 741-753. 
de este vínculo son: imitación, predisposición a la agresión, esquemas normativos a favor de la violencia o la agresión y alteraciones paulatinas en la cognición social.

En resumen, los trabajos revisados explican el contagio de las conductas criminales por medio de procesos psicológicos, dejando a los procesos o mecanismos externos al individuo con una presencia marginal. Así pues, en la investigación sobre el contagio de la violencia las dimensiones sociológicas del contagio, la transmisión o la dispersión de la violencia todavía no están estudiadas con suficiencia.

Además de la importancia que tiene la difusión contagiosa para el enfoque epidemiológico de la prevención, también existe interés académico por estudiar los patrones de difusión que tienen las conductas criminales en un espacio y tiempo determinados. Esta línea de investigación tiene que ver con la identificación de distintos tipos de difusión y su vinculación con otro tipo de procesos sociales desde el análisis espacial de la criminalidad. ${ }^{11}$ Los primeros trabajos que comenzaron a estudiar el tema mediante la aplicación técnicas de análisis espacial ${ }^{12}$ se han concentrado en dos puntos:

11 También hay buenos ejemplos de estudios sobre el contagio del crimen con otro tipo de técnicas como Jens Ludwig y Jeffrey R. Kling, "Is Crime Contagious?", Journal of Law and Economics, vol. 50, núm. 3, pp. 491-518, 2007, en doi:10.1086/ 519807

12 Baller, Anselin, Messner y Deane, "Structural Covariates of US County Homicide Rates: Incorporating Spatial Effects", Criminology, vol. 39, 2001, pp. 561-590; Messner, Anselin, Baller, Hawkins, Deane y Tolnay, "The Spatial Patterning of County Homicide Rates: An Application of Exploratory Spatial Data Analysis", Journal of Quantitative Criminology, vol. 15, núm. 4, 1999, pp. 423-450; Morenoff y Sampson, "Violent Crime and the Spatial Dynamics of Neighborhood Transition: Chicago, 1970-1990”, Social Forces, vol. 76, núm. 1, 1997, pp. 31-64, en doi:10.2307/2580317; Rosenfeld, Bray y Egley, "Facilitating Violence: A Comparison of Gang-Motivated, Gang-Affiliated, and Nongang Youth Homicides", Journal of Quantitative Criminology, vol. 15, núm. 4, 1999, pp. 495-516, en doi:10.1023/A:1007548309620; Smith, Frazee y Davison, "Furthering the Integration of Routine Activity and Social Disorganization Theories: Small Units of Analysis And the Study of Street Robbery As A Diffusion Process", Criminology, vol. 38, núm. 2, 2000, pp. 489-524; Tolnay, Deane y Beck, "Vicarious Violence: Spatial Effects on Southern Lynchings, 1890-1919", American Journal of Sociology, vol. 102, núm. 3, 1996, pp. 788-815. Para los interesados en conocer con más detalle el análisis espacial del crimen, revísese los capítulos 2, 6 y 33 de 
identificar la espacialidad del fenómeno y los spillover effects, es decir, los efectos que puede tener la variación de un fenómeno en la variación del mismo fenómeno en áreas colindantes. También hay algunas aplicaciones de técnicas de análisis espacial y de modelación espacial para explicar la difusión contagiosa de la criminalidad. ${ }^{13}$ No todas las preguntas de investigación sobre el contagio han sido trabajadas con análisis espacial; por ejemplo, está el estudio de Fagan, Wilkinson y Davies, ${ }^{14}$ en donde se analizan los mecanismos de contagio a partir de modelos de regresión de efectos mixtos.

Para el caso de México, la investigación sobre la difusión de la criminalidad, y en especial del crimen violento, se ha ido incrementando de manera paulatina. El principal foco de atención se ha colocado en la violencia derivada de los enfrentamientos entre las Organizaciones para el Tráfico de Drogas (отD) -y otras formas de crimen organizado- y enfrentamientos con las fuerzas estatales de seguridad y militares. Las investigaciones que se han desarrollado se han concentrado en tres factores para explicar el incremento en la violencia: los factores políticos que incentivan la violencia, ${ }^{15}$

Petras, Masyn, Piquero y Weisburd, Handbook of Quantitative Criminology, Nueva York, Springer-Verlag, 2010.

${ }^{13}$ LaFree, Dugan, Xie y Singh, "Spatial and Temporal Patterns of Terrorist Attacks by ETA 1970 to 2007", Journal of Quantitative Criminology, vol. 28, núm. 1, 2011, pp. 7-29; Rey, Mack y Koschinsky, "Exploratory Space-Time Analysis of Burglary Patterns”, Journal of Quantitative Criminology, vol. 28, núm. 3, 2011, pp. 509531, en doi:10.1007/s10940-011-9151-9; Ye y Wu, "Analyzing the Dynamics of Homicide Patterns in Chicago: ESDA and Spatial Panel Approaches”, Applied Geography, vol. 31, núm. 2, 2011, pp. 800-807.

${ }^{14}$ Fagan, Wilkinson y Davies, "Social Contagion of Violence", en Flannery, Vazsonyi y Waldman (eds.), The Cambridge Handbook of Violent Behavior, Cambridge, Cambridge University Press, 2007, pp. 688-724.

${ }^{15}$ Dell, Trafficking Networks and the Mexican Drug War, 2014, en http:/ /scholar. harvard.edu/dell/publications / trafficking-networks-and-mexican-drug-war-0; Duran-Martinez, Criminals, Cops, and Politicians: Dynamics of Drug Violence in Colombia and Mexico, Providence, Brown University, 2013; Osorio, Hobbes on Drugs: Understanding Drug Violence in Mexico, Notre Dame, University of Notre Dame, 2013; Rios, How Government Structure Encourages Criminal Violence: The Causes of Mexico's Drug War, Cambridge, Harvard, 2012; Snyder y Duran-Martinez, "Does Illegality Breed Violence? Drug Trafficking and State-Sponsored Protection Rackets", Crime, Law and Social Change, vol. 52, núm. 3, 2009, pp. 253-273. 
la estrategia de seguridad ${ }^{16}$ y cambios en la disponibilidad de armas de fuego y en el mercado de drogas. ${ }^{17}$

Otra línea de investigación que ha ido ganando presencia es el estudio de las características espaciales de la criminalidad y la violencia en México. ${ }^{18}$ Algunos trabajos han aplicado estas técnicas para estudiar los patrones de difusión de la criminalidad. ${ }^{19}$ En comparación con lo que se ha realizado en otros países, todavía se sabe poco sobre la espacialidad del crimen y en particular sobre la existencia de patrones de difusión que puedan ser considerados como difusión contagiosa. Los trabajos de Valdivia son un gran avance, ya que por medio de modelos de regresión espacial detecta vínculos similares al contagio entre las tasas de homicidio

${ }^{16}$ Calderón, Díaz-Cayeros, Magaloni, Robles y Olarte, The Temporal and Spatial Dynamics of Violence in Mexico, 2012, en https://www.dropbox.com/s/ce0lhfs9unsuqa3/paper_policies_violence_Aug30.pdf

17 Castillo, Mejía y Restrepo, Scarcity without Leviathan: The Violent Effects of Cocaine Supply Shortages in the Mexican Drug War. Centre for Global Development - Working Papers, 2014; Dube, Dube y García-Ponce, "Cross-Border Spillover: U.S. Gun Laws and Violence in Mexico", American Political Science Review, vol. 107, núm. 3, 2013, pp. 397-417.

${ }^{18}$ Ingram, The Local Educational and Regional Economic Foundations of Violence: A Subnational, Spatial Analysis of Homicide Rates across Mexico's Municipalities, 2014, en http://www.wilsoncenter.org/publication/educational-regional-foundationsviolence; Vilalta, "How Exactly Does Place Matter in Crime Analysis? Place, Space, and Spatial Heterogeneity", Journal of Criminal Justice Education, vol. 24, núm. 3, 2012, pp. 290-315; Vilalta, "Anomia institucional, espacialidad y temporalidad en las muertes asociadas a la lucha contra la delincuencia organizada en México", Mexican Studies/Estudios Mexicanos, vol. 29, núm. 1, 2013, pp. 280-319, en doi:10.1525/msem.2013.29.1.280; Vilalta y Muggah, "Violent Disorder in Ciudad Juarez: A Spatial Analysis of Homicide", Trends in Organized Crime, vol. 17, núm. 3, 2014, pp. 161-180; Vilalta, "Un modelo descriptivo de la geografía del robo en la zona metropolitana del Valle de México”, Journal of Latin American Geography, vol. 8, núm. 1, 2009, pp. 55-78.

${ }^{19}$ Valdivia y Castro, "Gender Bias in the Convergence Dynamics of the Regional Homicide Rates in Mexico”, Applied Geography, vol. 45, núm. 0, 2013, pp. 280291; Valdivia, "Análisis espacial de la dinámica del homicidio de mujeres en México a nivel municipal. Identificación y explicación de patrones de convergencia y polarización territorial”, en Riquer y Castro (eds.), Estudio nacional sobre las fuentes, orígenes y factores que producen y reproducen la violencia contra las mujeres, México, Segob/ Conavim, 2012. 
municipales. ${ }^{20}$ En su trabajo más reciente, desarrolla y analiza la posibilidad de convergencia entre el homicidio masculino y femenino, revelando otro tipo de implicaciones de la espacialidad que no habían sido contempladas con anterioridad. ${ }^{21}$

Hasta aquí se ha esbozado un panorama que ubica las preguntas sobre el contagio o la difusión contagiosa del crimen en dos ámbitos: en el enfoque epidemiológico o basado en la salud y en el análisis espacial de la criminalidad. En el primero, gran parte de la base empírica está en la identificación de mecanismos de imitación a nivel individual. El análisis espacial aborda el tema de la difusión desde una perspectiva agregada, tratando de identificar la espacialidad del fenómeno y a través de ella diferenciar patrones de difusión espacial y temporal que pueden ser considerados como difusión contagiosa. De igual forma incorpora técnicas de modelación espacial que buscan descubrir los mecanismos y procesos sociales que hacen posible la emergencia de patrones específicos de difusión, como sería el caso de la difusión contagiosa.

Para el caso de México es necesario desarrollar la segunda perspectiva para tener en claro las características del fenómeno y para aportar elementos que orienten el diseño de mejores políticas públicas para la prevención. Es decir, políticas de prevención que no den por hecho una difusión contagiosa del crimen sin contar con la evidencia necesaria. ¿Cómo se tendría que estudiar el contagio y qué evidencia hace falta? En primera instancia se tiene que identificar la espacialidad del fenómeno en una unidad geográfica específica. Un segundo paso es la identificación, diferenciación y análisis de los patrones de dispersión para comprobar si existe una dispersión similar al contagio. Además, estos dos primeros pasos tienen que llevarse a cabo bajo un marco longitudinal para captar las transiciones a lo largo del tiempo. Finalmente, una vez identificada la espacialidad y los patrones de dispersión se estaría en posición de realizar un ejercicio de modelación para identificar la presencia de interacciones entre tiempo y espacio,

${ }^{20}$ Loc. cit.

${ }^{21}$ Valdivia y Castro, op. cit. 
incluyendo algunas variables criminogénicas que podrían estar relacionadas con los mecanismos que hacen posible el contagio de la violencia. De acuerdo con esta propuesta, este trabajo presenta los resultados de un análisis a partir de los primeros dos puntos.

\section{ANÁlisis ESPACIAL DE LAS TASAS DE HOMICIDIO}

\subsection{Métodos}

La primera parte del análisis tiene tres objetivos: hacer una descripción de la distribución de la tasa de homicidio en México, conocer el grado de espacialidad del fenómeno por medio de la I de Moran $^{22}$ e identificar por medio de los índices locales de autocorrelación (LISA) los conglomerados o clusters para todos los municipios del país y su variación a lo largo del tiempo. Para ello se aplicó un Análisis Espacial Exploratorio (ESDA) con el programa GeoDa. ${ }^{23}$

\subsection{Datos}

Se usó la tasa de homicidio intencional a nivel municipal por sexo para el periodo 1990-2010. Los datos provienen del sistema de estadísticas vitales del inegi. Para el análisis espacial se empleó la configuración de municipios de 2005. Todos los análisis fueron realizados con el programa GeoDa.

${ }^{22}$ La I de Moran es una medida de la intensidad de la autocorrelación en las áreas de estudio. Sus valores varían de -1 a +1 , mientras más cercano a 1 esté el indicador mayor será el nivel de autocorrelación espacial.

${ }^{23}$ Anselin, Syabri y Kho, "GeoDa: An Introduction to Spatial Data Analysis", Geographical Analysis, vol. 38, núm. 1, 2006, pp. 5-22; Anselin, Spatial Econometrics: Methods and Models, Dordrecht, Kluwer AP, 1988; Fischer, M. M. y A. Getis, Handbook of Applied Spatial Analysis: Software Tools, Methods and Applications, Berlín-Heidelberg, Springer, 2009. 


\subsection{La tasa de homicidio como fenómeno regional}

\subsubsection{Distribución espacial y valores atípicos}

El primer análisis se enfocó en identificar la presencia de valores atípicos espaciales y sus cambios a lo largo del tiempo. Los valores atípicos espaciales permiten detectar valores extremos pero en relación con un área geográfica en particular. Los resultados muestran que la distribución espacial de la tasa de homicidio a nivel municipal es un fenómeno muy regionalizado, es decir, con altas concentraciones espaciales en algunos municipios. Como ya se ha mostrado en otros estudios, son alrededor de $5 \%$ de los municipios del país los responsables de los cambios ascendentes en la tasa de homicidio. ${ }^{24}$ Sin embargo, esto no quiere decir que la distribución sea estable. Por el contrario, al analizar la distribución de los valores extremos durante el periodo dos elementos resaltan: el cambio en la regionalización de las tasas que mudan del sur del país hacia el norte y el occidente; y el drástico incremento de municipios con valores atípicos a partir de 2007. Para tener una idea de estas modificaciones, el mapa 1 muestra la distribución de la tasa de homicidio por cuartiles en 1990 y el mapa 2 la de 2010. La regla para identificar los valores extremos son las regiones que se separan 3 veces del valor del rango del primer y el tercer cuartil. ${ }^{25}$

Los mayores cambios se ubican en los municipios arriba del tercer cuartil y en los valores atípicos, es decir, las variaciones se registran en esos dos grupos. Las transiciones más claras son: transición del sur (Oaxaca y Guerrero) hacia el suroeste y occidente

${ }^{24}$ Echarri-Cánovas, "Homicidio", en C. J. Echarri-Cánovas (ed.), Panorama estadístico de la violencia en México, México, El Colegio de México, 2012, pp. 51-103.

${ }^{25}$ Para evitar la detección de valores atípicos espurios y mejorar la identificación de patrones regionales, se utilizó la opción de homogenización bayesiana (empirical bayes smoothing). La técnica permite computar una tasa ponderando con las tasas de las áreas vecinas, con lo cual se controlan las abruptas variaciones que hay de municipio a municipio tomando en cuenta la población en riesgo (Anselin, Exploring Spatial Data with GeoDaTM: A Workbook, Urbana, University of Illinois, 2005, pp. 99-101). 
(Michoacán) y expansión en zonas "tradicionales"26 (Sonora y Sinaloa) hacia la frontera (Tamaulipas).

Una de las principales características de la violencia criminal en México es la sobrerrepresentación de la tasa masculina sobre la femenina; esta característica también se puede ver en la distribución espacial. Los cambios en la tasa general responden a los cambios en la tasa masculina. ${ }^{27}$ Por el contrario, la tasa femenina tiene una distribución homogénea en todo el territorio y con modificaciones mínimas en el periodo. La tasa femenina se concentra en los estados del centro del país y del sureste, las variaciones se concentran en el cuartil 25-50 y los valores atípicos altos. Sin embargo, las tasas extremas son muy estables y se concentran en un promedio de 500 municipios.

\subsubsection{Autocorrelación espacial}

Esta particular distribución es un buen indicio de la existencia de espacialidad en el fenómeno. Para medir el grado de autocorrelación espacial entre las tasas de homicidio y las áreas geográficas de referencia se empleó el índice de autocorrelación de Moran, o I de Moran, y el índice local de autocorrelación espacial (LISA).

La autocorrelación espacial se define como la representación de la relación entre una variable observada en todas las unidades espaciales cercanas y una medida de proximidad geográfica. ${ }^{28} \mathrm{El}$ índice global muestra el grado de autocorrelación del fenómeno en todo el territorio, mientras que el índice local o Lisa sólo toma

${ }^{26}$ Uso la palabra tradicionales para hacer referencia a las zonas del país donde la tasa de homicidio ha mantenido niveles por arriba de la media nacional durante todo el periodo.

${ }^{27}$ Para más información sobre la sobrerrepresentación de los hombres como víctimas y perpetradores de violencia consúltese: wHO, Global Status Report on Violence Prevention 2014, World Health Organization, the United Nations Development Programme, and the United Nations Office on Drugs and Crime, 2014.

28 Cliff y Ord, "Spatial and Temporal Analysis: Autocorrelation in Space and Time”, Quantitative Geography: A British View, 1981, pp. 104-110. 


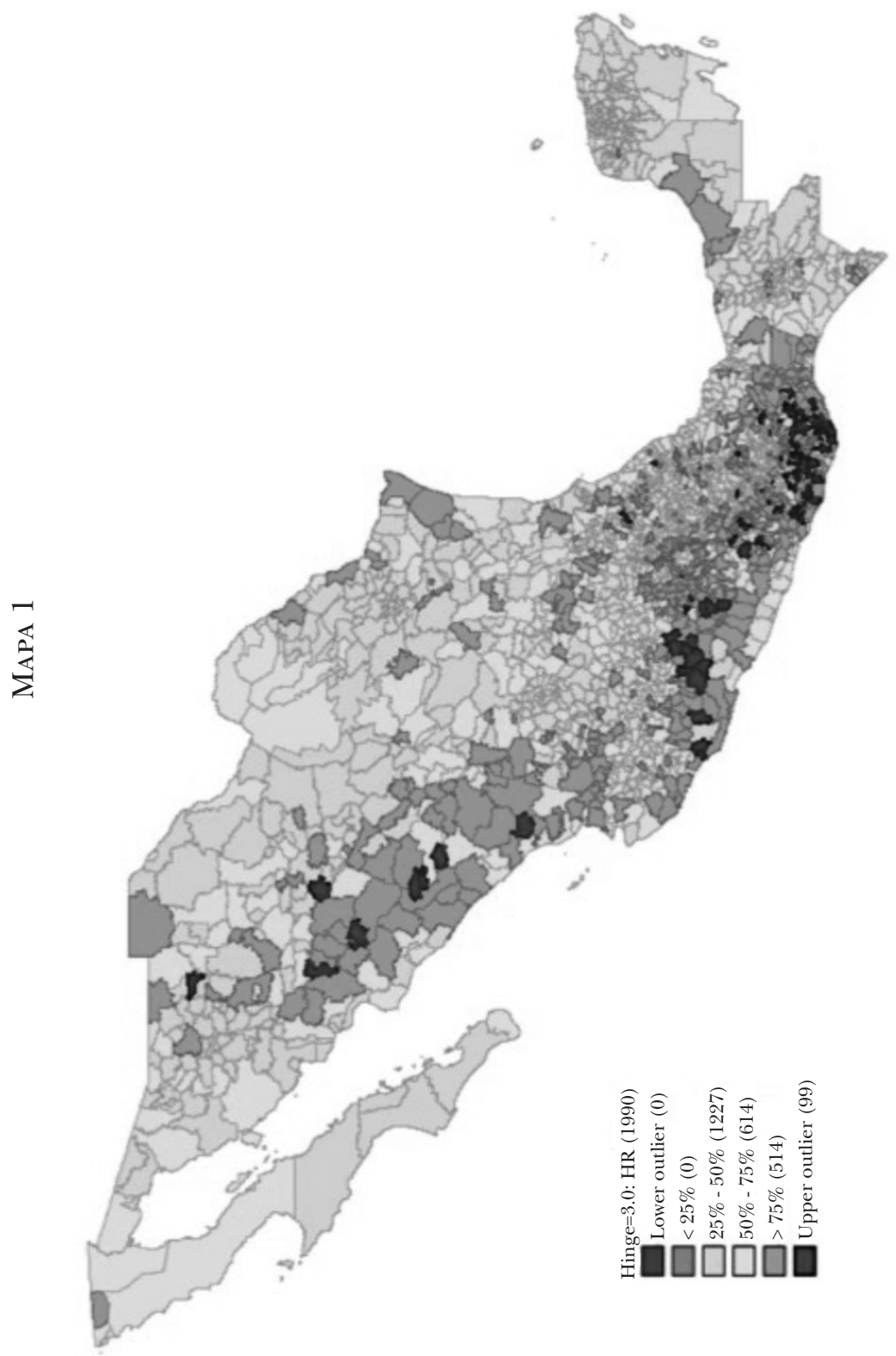




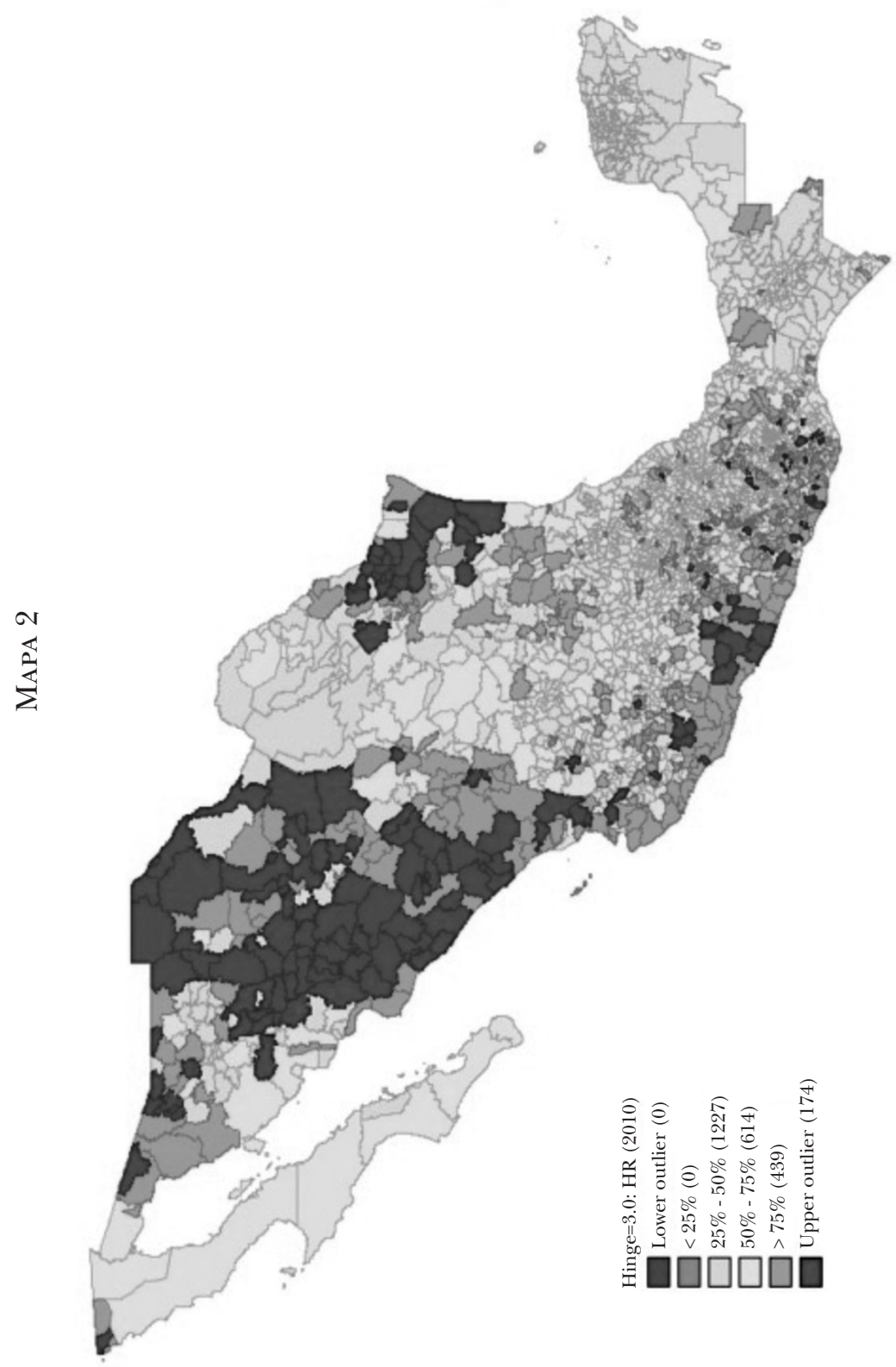


en cuenta el municipio de referencia. ${ }^{29}$ En otras palabras, el global es un promedio de todos los índices locales. Para el cómputo de los índices (global y local) de autocorrelación, GeoDa utiliza una aproximación numérica para probar el peso estadístico de los índices. A diferencia de la aproximación analítica, ésta no requiere de los supuestos de normalidad, pero tiene la desventaja de que los valores $p$ que genera dependen del número de permutaciones. Para el cómputo de los índices las permutaciones se emplean para conocer la probabilidad de un valor en una distribución bajo condiciones de aleatoriedad espacial. Para generar una distribución espacial aleatoria de referencia para el índice, se utiliza un set distinto de números aleatorios cada vez que se calcula el estadístico. Por esta razón los resultados no se pueden replicar con total precisión. En este análisis los índices global y local se realizaron con 999 permutaciones en cada uno de los cómputos. En el global todos los índices mostraron peso estadístico mayor a .001 con excepción de la tasa femenina de: $92,93,97,98,01$ y $10 .^{30}$

La gráfica 1 presenta los cambios en los niveles de autocorrelación de la tasa de homicidio a nivel municipal durante todo el periodo. ${ }^{31}$ ¿Qué implican estos resultados? Para la I de Moran, “el hecho de que exista autocorrelación espacial de la tasa de homicidios de mujeres y hombres a nivel municipal sugiere que pueden existir factores comunes o de interdependencia entre un municipio y sus vecinos que a su vez determinan la tasa de homicidio". ${ }^{32}$ Es decir, que la dimensión espacial de la tasa de homicidio cambia a lo largo del tiempo y por sexo. Sin embargo, también se puede

${ }^{29}$ Anselin, "Local Indicators of Spatial Association — LISA", Geographical Analysis, vol. 27, núm. 2, 1995, pp. 93-115.

${ }^{30}$ Esta falta de peso estadístico concuerda con los bajos niveles de autocorrelación espacial que tiene la distribución de la tasa femenina en el país.

${ }^{31}$ Otra decisión importante que se tiene que tomar antes de iniciar el ESDA son los criterios de vecindad. Geoda proporciona tres opciones para determinar los criterios de vecindad: distancias, matriz queen y matriz rook. Para este caso se eligió la matriz queen, ya que a diferencia de la rook no sólo toma en cuenta las áreas que comparten frontera, sino que también incluye a los vértices, es decir las diagonales (como el movimiento de la reina en el ajedrez).

32 Valdivia, "Análisis espacial de la dinámica del homicidio de mujeres en México a nivel municipal...”, p. 21. 


\section{GRÁFICA 1 \\ Autocorrelación espacial}

Tasa de homicidio a nivel municipal (1990-2010)

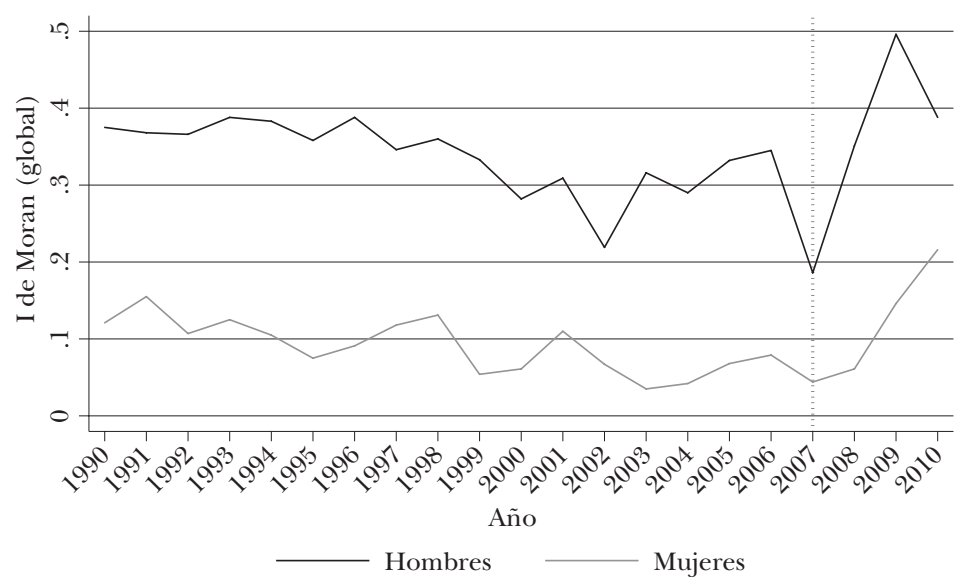

ver que la tasa de homicidios de mujeres está menos relacionada con factores espaciales que la tasa masculina; se trata de una distribución espacial diferenciada.

El índice global muestra el grado de regionalización del fenómeno, pero no dice nada sobre las diferencias internas. Por el contrario, el índice local (LISA) nos da información sobre los diferentes grados de autocorrelación local (entre los centros de los conglomerados y las variaciones de la variable dependiente en los municipios vecinos) y permite identificar dos tipos de autocorrelaciones. Primero están los valores positivos, también llamados conglomerados o hot spots. ${ }^{33}$ Estos incluyen grupos de tasas altas en el centro geográfico y en los municipios vecinos $(\mathrm{H}-\mathrm{H})$ y grupos de tasas

${ }^{33}$ Puesto que la distribución del homicidio en México es muy desigual ya que se acumula en aproximadamente una quinta parte de los municipios del país (en los años con las tasas más altas), además, dada la heterogeneidad poblacional de los municipios, las tasas son susceptibles a variaciones importantes. Para que esta característica no afecte el cálculo de la I de Moran y las LisA, se utilizó la estandarización bayesiana de tasas disponible en GeoDa (Assunção y Reis, "A New Proposal to Adjust Moran's I for Population Density”, Statistics in Medicine, vol. 18, núm. 16, 1999, pp. 2147-2162). 
bajas en el centro y en las áreas circundantes (L-L). En segundo lugar están los valores negativos o áreas atípicas donde las áreas centrales tienen valores distintos a las áreas que los rodean (H-L y L-H) ${ }^{34}$

Las zonas con valores positivos implican que los municipios que las componen tienen tasas de homicidio similares. Estos conglomerados pueden ser el resultado de un proceso de difusión contagiosa o un prerrequisito para observar el contagio. Por el contrario, las áreas atípicas pueden ser muestra de la ausencia de difusión ya que implican variaciones locales sin vinculación con la variación de las áreas vecinas. El mapa 3 presenta la distribución de municipios a partir de los valores LISA en 1990 y el mapa 4 para 2010. Para tener una idea de los cambios en los valores positivos de LISA durante el periodo, la gráfica 2 presenta la evolución de los índices de los conglomerados $\mathrm{H}-\mathrm{H}$.

GRÁFICA 2

Índice de autocorrelación del conglomerado H-H (1990-2010)

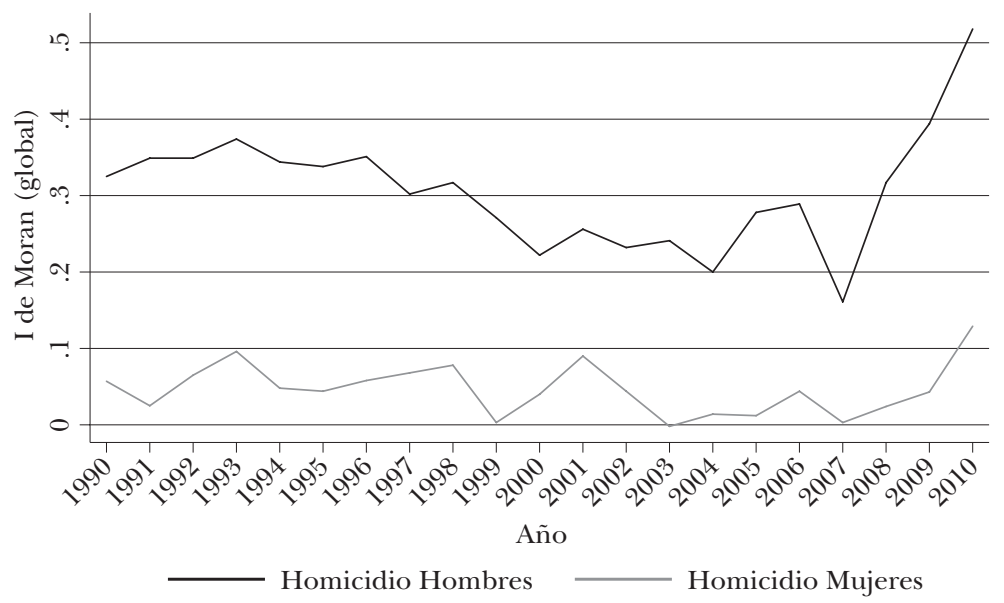

${ }^{34}$ Los valores atípicos son aquellos que se encuentran 1.5 veces por arriba del rango intercuartil o IQR, que es la diferencia entre el tercer cuartil (c3) y el primero (c1). El rango intercuartil describe el rango de la parte media de la distribución, ya que el $25 \%$ de los valores lo superan y un $25 \%$ está por debajo. 


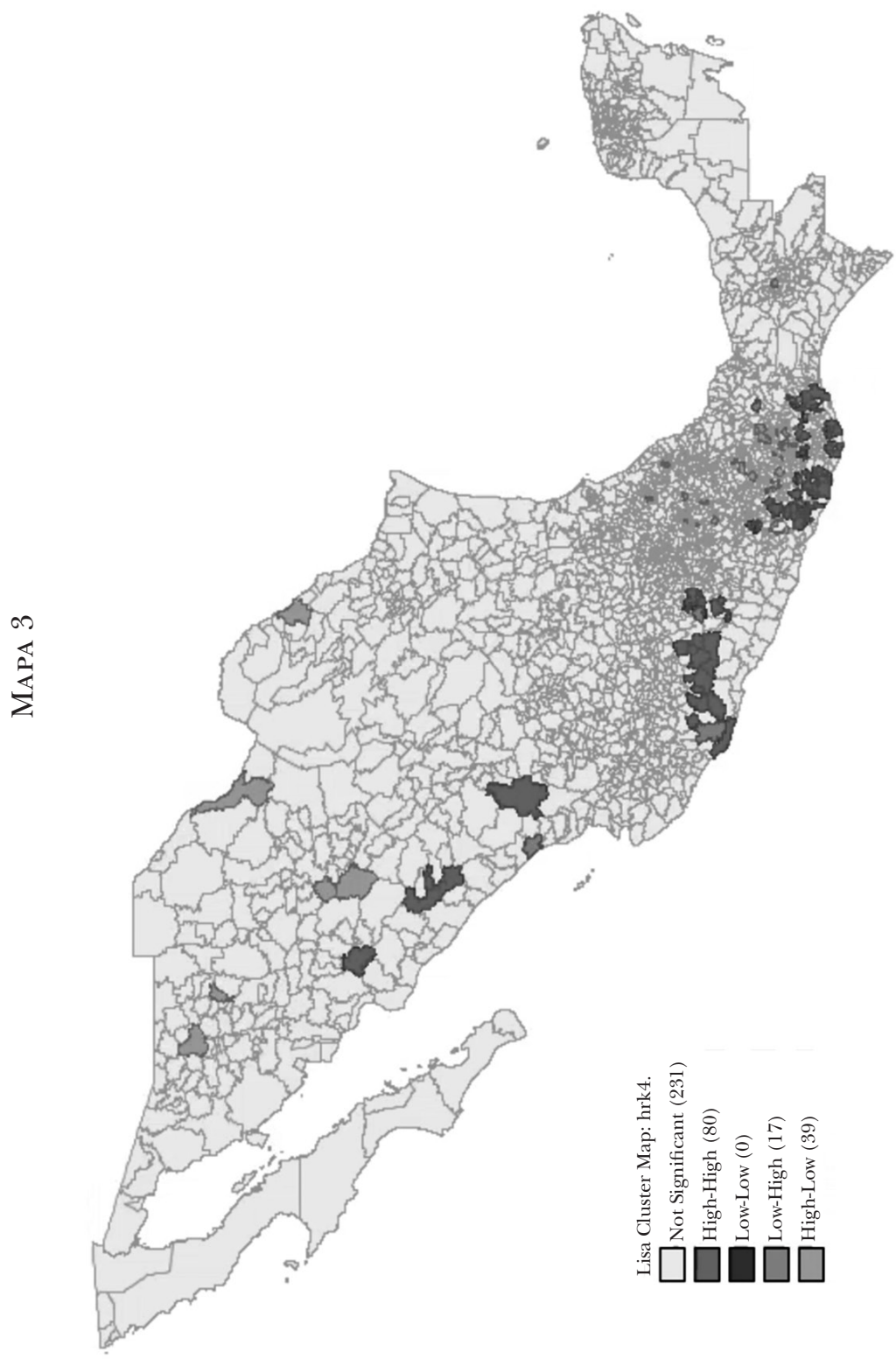




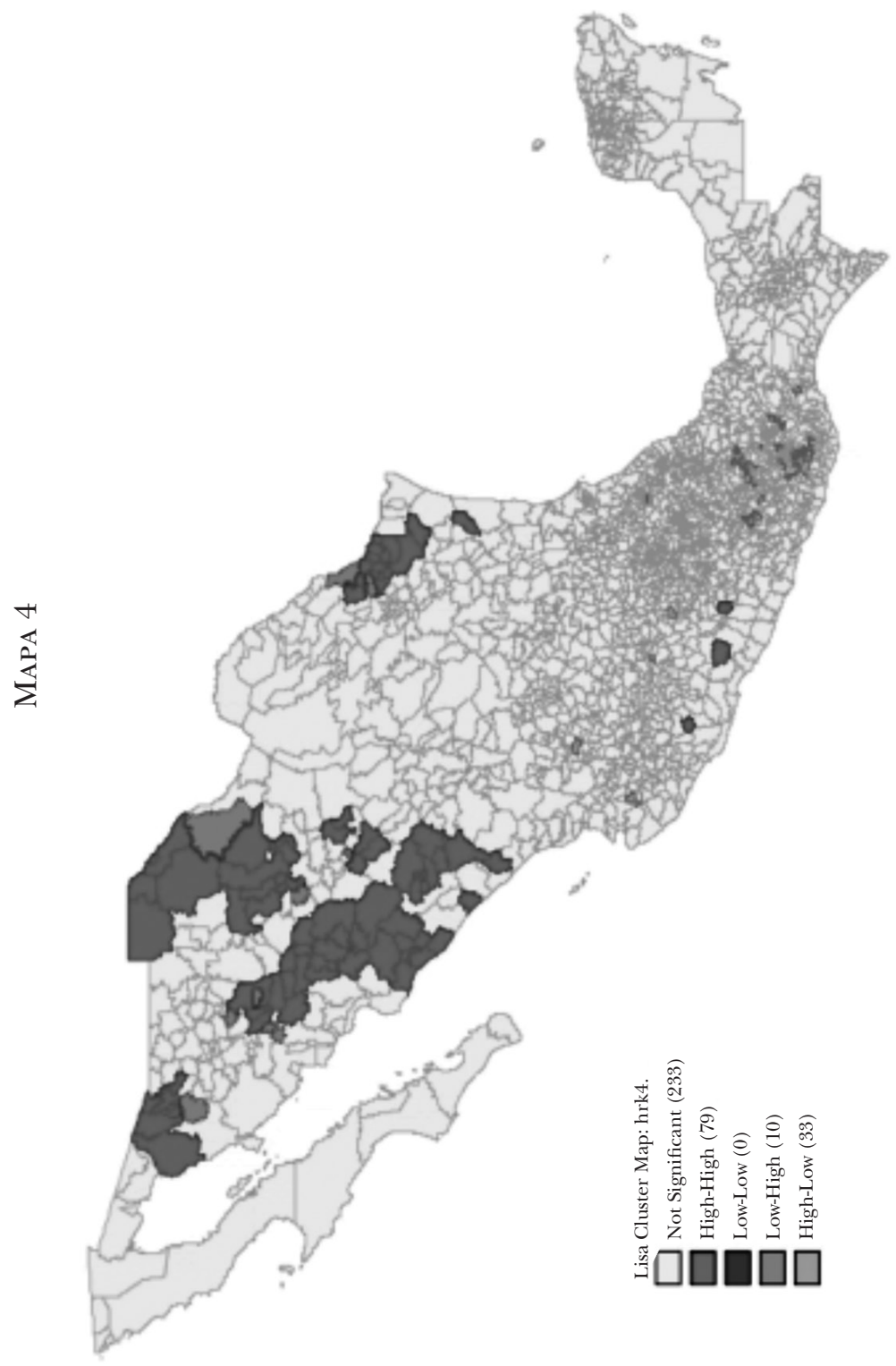


¿Qué podemos decir sobre los resultados de esta aproximación espacial a los datos? La dinámica de la distribución del homicidio intencional en el país es muy regional. Esto quiere decir que la distribución se concentra en un número menor de municipios (alrededor de 5\% de los municipios del país) y que el incremento en las tasas se debe al aumento en estos municipios. Pero las características del análisis espacial nos permiten identificar otras cosas. Los valores arrojados por los índices muestran que la regionalización del fenómeno es más que la concentración geográfica en una zona. En especial la autocorrelación local aporta elementos numéricos que indican que dicha regionalización puede estar asociada a un grupo de factores en común en los conglomerados de tipo H-H. Es decir, señalan la existencia de elementos espaciales que están relacionados con la variación en las tasas de homicidio. De igual forma también es posible ver que la espacialidad del fenómeno es variable a lo largo del tiempo y cambia de acuerdo al sexo (gráfica 2). Por ejemplo, la tasa masculina tiene variaciones importantes durante el periodo pero se mantiene dentro del mismo rango (con la excepción de 2010). Por el contrario, las variaciones de la tasa femenina se dan en un rango más amplio. Sin embargo, la diferencia en el número de municipios con valores Lisa positivos entre hombres y mujeres es reflejo de la sobrerrepresentación de la tasa masculina (véase tabla 1 y 2). Pero esta no es la única razón que explica esta diferencia; como se mencionó en párrafos anteriores, los valores positivos implican que la variación en la tasa de un área está relacionada con la variabilidad de la tasa de las áreas colindantes.

Por el contrario, los valores negativos o atípicos implican que dicho vínculo no existe. No obstante esto no quiere decir que no exista autocorrelación de las tasa con el área. En otras palabras, hay espacialidad, pero es un valor atípico puesto que no está estadísticamente vinculado con la variación de las áreas circundantes. Este es el caso particular de la tasa femenina a diferencia de la masculina. Si observamos el número de áreas en valores atípicos ( $\mathrm{L}-\mathrm{H}$ y H-L), salta a la vista que la mayoría de casos significativos en los hombres está en el conglomerado H-H mientras que para el caso de las mujeres la mayoría está en el grupo H-L. Con estos datos se 
puede suponer que de existir una difusión contagiosa sería más probable encontrarla en la tasa masculina y no en la femenina, pero se necesita otra aproximación para poder verificarlo.

\section{TABLA 1}

Municipios clasificados por LISA (tasa hombres)

\begin{tabular}{cccccc}
\hline & 1990 & 1995 & 2000 & 2005 & 2010 \\
\hline H-H & 82 & 79 & 43 & 47 & 90 \\
L-L & 0 & 0 & 0 & 0 & 0 \\
L-H & 19 & 18 & 19 & 26 & 10 \\
H-L & 47 & 41 & 57 & 55 & 40 \\
No Sig & 230 & 231 & 233 & 232 & 231 \\
\hline
\end{tabular}

TABla 2

Municipios clasificados por LISA (tasa mujeres)

\begin{tabular}{cccccc}
\hline & 1990 & 1995 & 2000 & 2005 & 2010 \\
\hline H-H & 8 & 4 & 5 & 0 & 18 \\
L-L & 0 & 0 & 0 & 0 & 0 \\
L-H & 23 & 21 & 19 & 25 & 20 \\
H-L & 143 & 143 & 155 & 140 & 114 \\
No Sig & 228 & 228 & 2227 & 228 & 230 \\
\hline
\end{tabular}

A manera de conclusión preliminar, este primer análisis de los datos nos ha permitido identificar algunas características de la distribución de la tasa de homicidio que justifican la necesidad de utilizar otro tipo de técnicas para verificar la presencia de una difusión de tipo contagiosa en los municipios del país. Ese es el objetivo de la segunda parte del trabajo.

\section{ANÁLISIS DE LOS PATRONES DE DISPERSIÓN}

Después de identificar la existencia de conglomerados significativos en la muestra, el siguiente paso para analizar los patrones de la 
dispersión es observar los cambios entre los niveles de dispersión y concentración. La gráfica 3 presenta la evolución de la tasa de concentración y el porcentaje de localidades con dispersión en un periodo de veinte años.

GrÁfICA 3

Dispersión y concentración del homicidio en México (1990-2010)

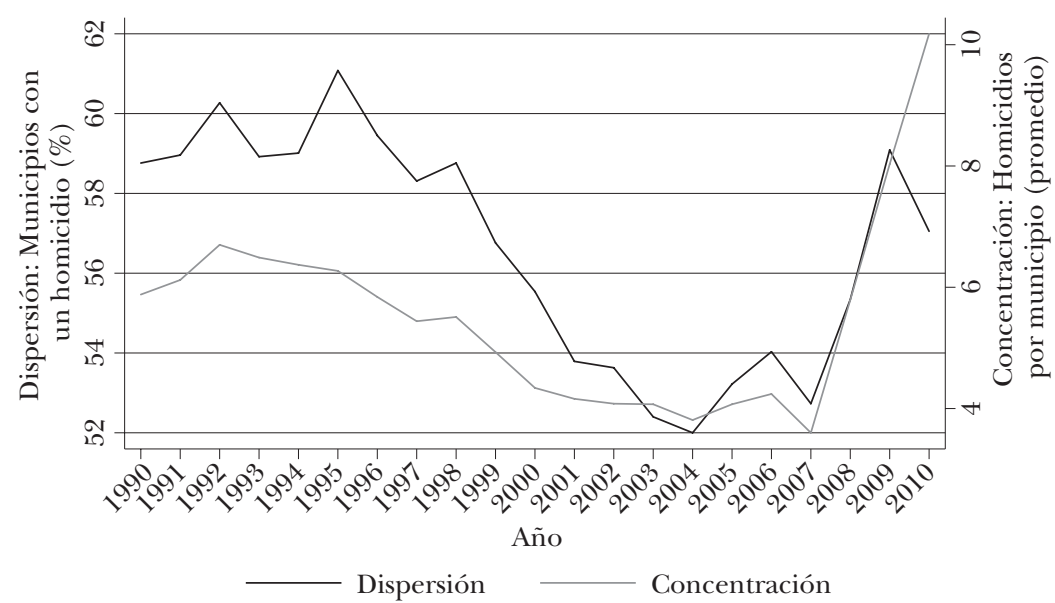

La gráfica muestra con claridad la dispersión del homicidio en alrededor de 50 y $60 \%$ de los municipios del país y sin grandes cambios durante el periodo. Por el otro lado, la concentración ha disminuido de manera constante desde 1992 hasta que en 2007 la tendencia se revirtió abruptamente. Esta combinación entre patrones relativamente estables de dispersión (a pesar del impacto de 2007) y el incremento en la concentración a partir de 2007 puede ser un indicio de que las áreas que recibieron la nueva concentración de homicidios estén dentro del rango de municipios que han sido parte de la dispersión histórica del homicidio en México. Esta idea cobra sentido si recordamos el análisis descriptivo en páginas anteriores. El cambio en la distribución de la tasa de homicidio del sur y suroeste del país hacia el centro y al occidente no implican un fenómeno de desplazamiento, es decir, a pesar 
de un cambio en la distribución las zonas "originales" seguían manteniendo tasas arriba de la media nacional. Además, también cabe recordar que zonas como el noroeste han mantenido tasas por arriba de la media nacional en todo el periodo. Ahora, si la dispersión se ha mantenido dentro de un mismo rango en los últimos veinte años y la concentración se triplicó con el efecto de 2007, vale la pena preguntarse si este cambio en la concentración responde a algún tipo de patrón más específico que la ocurrencia previa de homicidios.

Precisamente uno de los posibles patrones que vale la pena investigar es la difusión de tipo contagiosa. Para detectar los patrones que siguieron estos cambios hace falta un método que permita identificar tanto las modificaciones en la difusión espacial a lo largo del tiempo como su direccionalidad. Como se mencionó anteriormente, los métodos convencionales de análisis espacial son estáticos y no son aptos para detectar los cambios, por lo que es necesario buscar técnicas de análisis que permitan captar el cambio espacial a lo largo del tiempo. Sin embargo, sabemos que la mayoría de los estudios que han estudiado la difusión contagiosa no emplean técnicas espaciales. De los pocos ejemplos que utilizan análisis espacial, ninguno emplea una técnica que pueda dar cuenta de los cambios en el tiempo. Después de una amplia revisión de la bibliografía sólo se encontraron dos estudios sobre las tasas de homicidio que emplean técnicas de análisis espacial e incorporan la variable tiempo. Es el caso de los estudios de Jacqueline Cohen y George Tita para detectar y clasificar los procesos de difusión espacial. ${ }^{35}$ La investigación de Cohen y Tita propone un método para identificar y clasificar la difusión en áreas urbanas de la violencia juvenil (1999) y de los disparos de arma de fuego (2004). Su propuesta distingue entre la difusión contagiosa que se puede dar entre unidades colindantes y la difusión sin proximidad.

35 Cohen y Tita, "Diffusion in Homicide: Exploring a General Method for Detecting Spatial Diffusion Processes", Journal of Quantitative Criminology, vol. 15, núm. 4, 1999, pp. 451-493; Tita y Cohen, "Measuring Spatial Diffusion of Shots Fired Activity Across City Neighborhoods", Spatially Integrated Social Science, 2004, pp. 171-204. 


\subsection{Método}

La idea de Cohen y Tita es elaborar un método para identificar transiciones y diferenciar tipos de difusión, partiendo de la idea de que una epidemia implica cambios no monótonos, definidos por cambios abruptos de crecimiento acelerado, seguido de un periodo de disminuciones paulatinas. Para que este patrón temporal sea posible tiene que existir un mecanismo de transmisión del agente infeccioso entre los individuos de una población. Tomando esto en cuenta, si observamos la distribución de la tasa de homicidio en el país, podemos reconocer que la tendencia ha seguido un comportamiento no lineal y que se ha concentrado tanto en algunas zonas como en algunos subgrupos poblacionales. La tarea ahora es primero identificar patrones y posteriormente identificar los mecanismos de difusión y entender los procesos sociales que influyen en estos eventos.

Para identificar los patrones se entiende a la difusión como el proceso general de movimiento y al contagio como el mecanismo que hace posible dicho movimiento. A partir de esta idea se conciben dos tipos de difusión: expansión y traslado. Expansión tiene que ver con la difusión a partir de un origen, donde el punto de origen suele mantener los mismos niveles de incidencia criminal. El traslado es el tipo de difusión que parte desde un punto de origen, como un incendio que se mueve por entre un territorio buscando nuevas fuentes de combustión. Para el caso del crimen este tipo de difusión se conoce como desplazamiento. Un segundo mecanismo para la expansión del homicidio es la difusión jerárquica. Este proceso implica la transmisión a través de una secuencia ordenada de clases o lugares. Un ejemplo es la transmisión de ideas o estilos de zonas metropolitanas a áreas de menor tamaño, todo esto sin necesitar que estén conectados entre sí o que exista proximidad geográfica. ${ }^{36}$

Ahora bien, para poder detectar y discriminar entre patrones se propone un análisis basado en el análisis espacial exploratorio o exploratory spatial data analysis (ESDA) para identificar

${ }^{36}$ Cohen y Tita, "Diffusion in Homicide...". 
los conglomerados espaciales a lo largo de un territorio. El método consta de los siguientes pasos. En primer lugar se tienen que calcular los valores LISA locales para cada municipio. Con estos valores se clasifican los conglomerados con peso estadístico en cuatro categorías: HH (alto-alto), LL (bajo-bajo), HL (alto-bajo) y LH (bajo-alto). ${ }^{37}$ Una vez que son generados los valores LisA también se tiene la tasa de homicidio local estandarizada (núcleo) y la tasa ponderada de las localidades vecinas. A partir de estas tasas, año con año se pueden detectar y medir las transiciones entre las parejas de conglomerados. Cada una de estas transiciones se clasifica de acuerdo a su conglomerado en $t$ y en $t+1$, dando como resultado grupos como: $n_{11}, n_{12}, n_{21}, n_{22}$. El grupo $n_{11}$ indica que los valores de $\mathrm{n}_{1}$ en t no cambiaron en $t+1$ mientras que en $\mathrm{n}_{12}$ sí hay una transición (en este caso incremental) de $n_{1}$ en $t$ a $n_{2}$ en $t+1$.

Ahora que ya se tiene detectado si existe o no una transición, el paso que sigue es verificar si la transición es estadísticamente diferente al resto. Para ello, la nueva clasificación de parejas de conglomerados sirve para identificarlas como un punto de referencia en un espacio euclidiano bidimensional y así calcular las distancias euclidianas entre las unidades en $t$ y en $t+1$. Las distancias tendrán peso estadístico si sus valores son mayores a dos desviaciones estándar con respecto a la media. Una vez identificados los pares significantes y tomando en cuenta el origen de la difusión es posible identificar el tipo de difusión.

La tabla 3 muestra la clasificación completa de pares en $t$ y $t+$ 1. A partir de esta tabla, Cohen y Tita identifican cuatro posibles tipos de transiciones: incremento jerárquico $\left(n_{12}, n_{13}\right.$ y $\left.n_{14}\right)$, reducción jerárquica $\left(n_{41}, n_{42}, \mathrm{n}_{43}\right)$, incremento contagioso $\left(n_{23}, n_{24}, n_{34}\right.$, $\left.n_{32}\right)$ y reducción contagiosa $\left(n_{21}, n_{31}, n_{32}, n_{23}\right)$. En la diagonal $\left(n_{11}\right.$, $n_{22}, n_{33}$ y $\left.n_{44}\right)$ no hay cambio entre $t$ y $t+1$. La diferencia entre las

${ }^{37}$ Los conglomerados implican grupos de áreas colindantes. Las etiquetas y los mapas que genera el software sólo señalan un municipio; sin embargo, el área se considera como el núcleo del conglomerado, es decir un área que se encuentra rodeada de municipios con altas tasas de homicidio. La técnica identifica la variabilidad espacial conjunta de las áreas colindantes; de ahí provienen las cuatro categorías HH (núcleo alto rodeado de localidades altas), LL (núcleo bajo rodeado de localidades bajas), etc. 
transiciones contagiosas y las jerárquicas está en que las primeras se dan entre localidades conectadas y las transiciones jerárquicas se dan entre localidades no conectadas. Esta forma de clasificar las transiciones también permite diferenciar si el área local (o núcleo) es el resultado de la difusión o es el generador de la difusión.

TABLA 3

Posibles transiciones longitudinales entre áreas colindantes

\begin{tabular}{ccccc}
\hline $\begin{array}{c}\text { Pares núcleo- } \\
\text { vecino en } \mathrm{t}\end{array}$ & \multicolumn{5}{c}{ Cambio en pares núcleo-vecino en $\mathrm{t}+$ 1 $^{*}$} \\
\hline $\mathrm{LL}$ & $\mathrm{n}_{11}$ & $\mathrm{n}_{12}$ & $\mathrm{n}_{123}$ & $\mathrm{n}_{14}$ \\
$\mathrm{HL}$ & $\mathrm{n}_{21}$ & $\mathrm{n}_{22}$ & $\mathrm{n}_{23}$ & $\mathrm{n}_{24}$ \\
LH & $\mathrm{n}_{31}$ & $\mathrm{n}_{32}$ & $\mathrm{n}_{33}$ & $\mathrm{n}_{34}$ \\
HH & $\mathrm{n}_{41}$ & $\mathrm{n}_{42}$ & $\mathrm{n}_{43}$ & $\mathrm{n}_{44}$ \\
\hline
\end{tabular}

* cambios con significancia estadística

Con esta información también se pueden calcular dos tipos de tasas. La tasa de difusión que mide la prevalencia de un tipo de difusión sobre otra y la tasa de difusión de efectos cruzados que mide la relación entre el homicidio de un tipo en una localidad y la probabilidad de movimiento en el homicidio de un tipo distinto en otra localidad (p. ej. la tasa de homicidio masculina en el municipio $x$ en $t$ afecta la tasa de homicidio femenina en el municipio y en $t+1)$.

\subsection{Resultados}

Una vez creadas las clasificaciones podemos obsevar que, con respecto al total de municipios clasificados en el periodo (véase la tabla 4), la pareja más frecuente es LL (bajo-bajo) con 57\%.$^{38}$ Esto tiene sentido si recordamos que en nuestra descripción inicial de

${ }^{38} \mathrm{El}$ grupo LL forma parte de los pares de conglomerados que se encuentran en la diagonal (tabla 3), es decir son los municipios estacionarios. 
la distribución de la tasa de homicidio se define tanto por la presencia de valores atípicos como por la gran regionalización del fenómeno. Es decir, este resultado concuerda con el hecho de que la mayoría de los municipios del país tienen tasas por debajo de la media nacional. El segundo lugar lo ocupa el par HH (alto-alto) en la tasa masculina $(32.3 \%$ ) y el tercer lugar lo tiene el par LH $(17 \%)$ en la tasa femenina. Sin embargo, en el conteo de las áreas con cambios significantes las transiciones HH para hombres y LH para mujeres siguen teniendo mayor presencia, pero su porcentaje se reduce de manera importante $7.3 \%$ ( $\mathrm{HH}$ ) y $2 \%(\mathrm{LH})$. La tabla 4 también muestra que del total de pares ubicados en нн (1804) sólo 22.2\% son transiciones significativas y para LH apenas supera $19 \%$. Esta primera aproximación permite notar que en términos relativos las transiciones detectadas son más frecuentes que el porcentaje de municipios que suelen explicar las alzas en la tasa nacional. Con todo, hasta aquí no hemos diferenciado por tipo de transiciones para saber cómo se distribuyen dentro de ese porcentaje. Para saber esto es necesario calcular las tasas de difusión para las cuatro transiciones posibles.

La tasa de difusión es la proporción de un tipo de transición con respecto al resto de transiciones sin contar los pares clasificados como estáticos. Para saber si la tasa de difusión es diferenciable se calcula una tasa de comparación tomando como numerador al resto de transiciones en la misma dirección y como denominador al total de transiciones sin las estáticas. Con esto, los cocientes de difusión resultantes para cada tipo son sometidos a una prueba $t$ de comparación de medias para saber si la tasa es estadísticamente diferente a la tasa de comparación.

¿Qué necesitamos encontrar para observar la difusión contagiosa del homicidio? De acuerdo con la investigación de Cohen y Tita, tenemos que identificar un exceso de transiciones significantes el tipo LH en thacia HL o HH en $t+1 .{ }^{39}$ Es decir, contagio entre vecinos con el núcleo como origen y contagio entre vecinos con el núcleo como resultado. Tomando en cuenta las transiciones de todo el periodo, los resultados de la tasa general (tabla 5) muestran

${ }^{39}$ Cohen y Tita, "Diffusion in Homicide...", p. 474. 
que la transición con mayor prevalencia es el incremento entre vecinos con el núcleo como producto (.256) y la disminución jerárquica con el núcleo como producto.

\section{TABLA 4}

Pares núcleo-vecino de la tasa de homicidio en México (1990-2010)

\begin{tabular}{|c|c|c|c|c|c|c|c|c|}
\hline & \multicolumn{2}{|c|}{$H H$} & \multicolumn{2}{|c|}{$H L$} & \multicolumn{2}{|c|}{$L H$} & \multicolumn{2}{|c|}{$L L$} \\
\hline \multicolumn{9}{|c|}{ Frecuencias en todas las áreas } \\
\hline & \# & $\%$ & $\#$ & $\%$ & $\#$ & $\%$ & $\#$ & $\%$ \\
\hline General & 1804 & 32.8 & 193 & 3.5 & 380 & $6.9 \%$ & 3116 & $56.7 \%$ \\
\hline Hombres & 1819 & 32.3 & 197 & 3.5 & 409 & $7.3 \%$ & 3215 & $57.0 \%$ \\
\hline Mujeres & 331 & 8.9 & 372 & 10.0 & 631 & $17.0 \%$ & 2386 & $64.1 \%$ \\
\hline \multicolumn{9}{|c|}{ Frecuencias de áreas significantes } \\
\hline General & 400 & 7.3 & 2 & 0.0 & 73 & $2.0 \%$ & 0 & $0.0 \%$ \\
\hline Hombres & 416 & 7.4 & 3 & 0.1 & 79 & $1.4 \%$ & 1 & $0.0 \%$ \\
\hline Mujeres & 31 & 0.8 & 3 & 0.1 & 75 & $2.0 \%$ & 6 & $0.2 \%$ \\
\hline \multicolumn{9}{|c|}{ Porcentaje de áreas significantes por conglomerado } \\
\hline General & & 22.2 & & 1.0 & & 19.2 & & 0.0 \\
\hline Hombres & & 22.9 & & 1.5 & & 19.3 & & 0.0 \\
\hline Mujeres & & 9.4 & & 0.8 & & 11.9 & & 0.3 \\
\hline
\end{tabular}

En las tablas 6 y 7 se muestran los resultados para la tasa de homicidio por sexo. Para la tasa masculina tiene mayor presencia el incremento entre zonas colindantes con el núcleo como producto (.311) así como la disminución jerárquica con la misma dirección (.350). La tasa femenina (tabla 7) tiene una difusión contagiosa con el núcleo como producto con una frecuencia menor que la tasa de difusión general y de hombres (.117). Por el otro lado, a pesar de que la disminución jerárquica tiene un cociente similar a las anteriores tasas, ésta no es estadísticamente diferenciable con el resto de transiciones. ${ }^{40}$

40 Para tener una mejor idea de las implicaciones de la distribución de las tasas a partir de la clasificación, vale la pena recordar que el tipo de difusión que 
TABla 5

Patrones de cambios anuales en áreas núcleo y locales Tasa de homicidio en México (1990-2010)

\begin{tabular}{lcc}
\hline & \multicolumn{2}{c}{$\begin{array}{c}\text { Tasa de áreas con cambios significantes } \\
\text { en el siguiente año }\end{array}$} \\
\cline { 2 - 3 } \multicolumn{1}{c}{ Tipo de difusión } & Difusión & Otras \\
\hline Núcleo como resultado & & \\
\hline Incremento jerárquico & 0.000 & 0.089 \\
Incremento contagioso & $0.256^{* *}$ & 0.000 \\
Disminución contagiosa & 0.021 & 0.136 \\
Disminución jerárquica & $0.314^{* *}$ & 0.005 \\
\hline Núcleo como origen & & \\
\hline Incremento jerárquico & 0.000 & $0.23 * *$ \\
Incremento contagioso & 0.000 & $0.224 * *$ \\
Disminución contagiosa & 0.000 & 0.004 \\
Disminución jerárquica & 0.000 & 0.005 \\
\hline$* p<.05$ & $* * p<.01$ & $* * * p<.001$
\end{tabular}

La otra tasa que se puede calcular con estos datos es la de efectos transversales. Con ella, se puede identificar si la prevalencia de una transición se da entre dos tipos distinto de homicidio. En este caso se trata de ver si un tipo de transición en un tipo de homicidio (hombres) influye en la variación de otro tipo de homicidio (mujeres). La tabla 8 muestra que el incremento contagioso con el núcleo como resultado sigue teniendo la mayor prevalencia en los dos tipos de efectos transversales. Sin embargo, es más recurrente el efecto hombre a mujer (.375) que mujer a hombre. Finalmente la disminución jerárquica también presenta un peso similar a las otras tasas de difusión en los efectos transversales.

predomina en el territorio es la que afecta al núcleo, es decir, son los incrementos en las zonas vecinas las que determinan el incremento en el núcleo. En este caso los municipios identificados representan a los núcleos que resultaron de este tipo de difusión, por lo que los municipios circundantes también tienen tasas altas de homicidio. 
TABla 6

Patrones de cambios anuales en áreas núcleo y locales Tasa de homicidio masculina en México (1990-2010)

\begin{tabular}{lcc}
\hline & \multicolumn{2}{c}{ Tasa de áreas con cambios significantes } \\
& \multicolumn{1}{c}{ Tipo el siguiente año } \\
\cline { 2 - 3 } Nifusión & Difusión & Otras \\
\hline Núcleo como resultado & & \\
\hline Incremento jerárquico & 0.000 & 0.116 \\
Incremento contagioso & $0.311^{* *}$ & 0.000 \\
Disminución contagiosa & 0.021 & 0.130 \\
Disminución jerárquica & $0.350^{* *}$ & 0.005 \\
\hline Núcleo como origen & & \\
\hline Incremento jerárquico & 0.000 & $0.256^{* *}$ \\
Incremento contagioso & 0.000 & $0.245^{* *}$ \\
Disminución contagiosa & 0.000 & 0.004 \\
Disminución jerárquica & 0.000 & 0.005 \\
$* p<.05$ & $* * p<.01$ & $* * * p<.001$
\end{tabular}

TABla 7

Patrones de cambios anuales en áreas núcleo y locales Tasa de homicidio femenina en México (1990-2010)

\begin{tabular}{lcc}
\hline & \multicolumn{2}{c}{$\begin{array}{c}\text { Tasa de áreas con cambios significantes } \\
\text { en el siguiente año }\end{array}$} \\
\cline { 2 - 3 } \multicolumn{1}{c}{ Tipo de difusión } & Difusión & Otras \\
\hline Núcleo como resultado & & \\
\hline Incremento jerárquico & 0.000 & 0.020 \\
Incremento contagioso & $0.117 * * *$ & 0.000 \\
Disminución contagiosa & 0.016 & 0.034 \\
Disminución jerárquica & $0.227 * * *$ & 0.012 \\
\hline Núcleo como origen & & \\
\hline Incremento jerárquico & 0.000 & $0.07 * * *$ \\
Incremento contagioso & 0.000 & $0.059 * *$ \\
Disminución contagiosa & 0.000 & 0.006 \\
Disminución jerárquica & 0.000 & 0.006 \\
\hline$* p<.05$ & $* * p<.01$ & $* * * p<.001$
\end{tabular}


TABLA 8

Patrones de cambios anuales en áreas núcleo y locales Tasa de homicidio en México (1990-2010). Efectos transversales

\begin{tabular}{lllll}
\hline \multicolumn{1}{c}{ Hombre/Mujer } & \multicolumn{2}{c}{ Mujer/Hombre } \\
\hline \multicolumn{1}{c}{ Tipo de difusión } & Difusión $\mid$ & Otras & Difusión $\mid$ & Otras \\
\hline Núcleo como resultado & & & \\
\hline Incremento jerárquico & 0.000 & 0.020 & 0.000 & $0.117^{*}$ \\
Incremento contagioso & $0.375^{* * *}$ & 0.000 & $0.19 * *$ & 0.000 \\
Disminución contagiosa & 0.000 & $0.189 *$ & 0.000 & 0.006 \\
Disminución jerárquica & $0.296^{* *}$ & 0.000 & $0.125^{*}$ & 0.000 \\
\hline Núcleo como origen & & & & \\
\hline Incremento jerárquico & 0.000 & $0.251^{* *}$ & 0.000 & $0.122^{* *}$ \\
Incremento contagioso & 0.000 & 0.187 & 0.000 & $0.14^{* *}$ \\
Disminución contagiosa & 0.000 & 0.005 & 0.000 & 0.000 \\
Disminución jerárquica & 0.004 & 0.000 & 0.000 & 0.000 \\
\hline$* p<.05$ & $* * p<.01$ & $* * * p<.001$ &
\end{tabular}

¿Qué características tienen los municipios detectados? La tabla 9 muestra algunos descriptivos de los municipios que fueron clasificados en los cuatro tipos de transiciones detectadas. La difusión más frecuente es el incremento contagioso con mayor presencia en la tasa general y en la tasa de hombres; para la tasa de mujeres sólo se detectaron dos áreas con este tipo de transición. El otro tipo de transición es la disminución jerárquica también con mayor prevalencia en la tasa general y en la tasa de hombres. La tasa de mujeres tiene el menor número de transiciones jerárquicas, pero son más frecuentes que el incremento contagioso. Otra diferencia es la magnitud de las tasas de los municipios que integran los grupos. Los municipios de incremento contagioso tienen una tasa de homicidio más homogénea y con una media debajo de la nacional. Por el contrario, los municipios de disminución jerárquica son más heterogéneos y tienen una media muy por arriba del promedio nacional. Con esto se puede decir que la mayoría de los incrementos contagiosos detectados se han dado a partir de tasas por debajo de la media nacional. Por el contrario, las disminuciones se dan a partir de tasas por arriba de la media. 
TABla 9

Descriptivos por tipo de transición

\begin{tabular}{|c|c|c|c|c|c|c|}
\hline \multicolumn{3}{|c|}{ Transición * } & $\mathrm{N}$ & Media & Rango & Varianza \\
\hline Difusión contagiosa & $(+)$ & $(G)^{1}$ & 21 & 1.57 & 20.72 & 26.29 \\
\hline Difusión contagiosa & $(+)$ & $(\mathrm{H})$ & 29 & 2.84 & 31.92 & 51.55 \\
\hline Difusión contagiosa & $(+)$ & $(\mathrm{F})$ & 2 & 0.00 & 0.00 & 0.00 \\
\hline Difusión jerárquica & $(-)$ & (G) & 33 & 106.09 & 174.45 & 2632.13 \\
\hline Difusión jerárquica & $(-)$ & $(\mathrm{H})$ & 35 & 197.02 & 362.78 & 10510.99 \\
\hline Difusión jerárquica & $(-)$ & $(\mathrm{F})$ & 5 & 25.70 & 43.56 & 293.64 \\
\hline
\end{tabular}

* El método empleado para detectar la transición toma en cuenta el cambio de $t$ a $t+1$ pero el municipio que identifica es el municipio en t. Por ello los descriptivos reportados son de los municipios en t. Este es el caso de los dos municipios con difusión contagiosa en la tasa de mujeres, tenían una tasa de 0 en $t$ pero se detectó un incremento de $t+1$.

${ }^{1}(+)$ incremento, (-) disminución, $(\mathrm{G})$ tasa general, $(\mathrm{H})$ tasa hombres, $(\mathrm{M})$ tasa mujeres.

Un resultado sorpresivo es que, al revisar los años en que se registraron las transiciones, no parece existir relación entre los dos grandes puntos de inflexión en la tendencia del homicidio y modificaciones en la frecuencia de las transiciones. Estos dos puntos son 1992 (la tasa general pasó de superar los 20 homicidios por cada 100000 personas en 1992 hasta alcanzar los 10 homicidios en 2007) y 2007 (la tasa se recupera en tres años y supera los 20 homicidios). De los 11 años en donde se registró este tipo de difusión, 17 se encuentran entre 1992 y 2005, mientras que sólo se registraron 4 de 2007 a 2009. La difusión en la tasa de hombres se dio a lo largo de 15 años, 22 transiciones de 1992 a 2006 y 7 de 2007 a 2009. En periodos de tres años se dio el mismo número de transiciones (7) en 95,97 y 99 que de 2007 a 2009. En el caso de la tasa de mujeres estas se dan en 2008 y 2009. Para el caso de la disminución jerárquica la mayoría de las transiciones se dan entre 1990 y 2005 (14 años) y 5 en 2007 y 2008. El caso de la tasa de hombres se distribuye en 16 años con 30 entre 1990-2005 y 5 en 2007-2008. Los cambios en la tasa de mujeres se limitan a cinco años con cinco transiciones entre 1990 y 2006. 
La distribución espacial de las transiciones es coherente con los cambios en la distribución geográfica de la tasa de homicidio. Para la tasa general, los estados con más transiciones con incremento están en Oaxaca (13), Chihuahua (4), Sonora (2), Durango (1) y Guerrero (1). La distribución cambia un poco con la tasa masculina: Oaxaca (13), Chihuahua (8), Sonora (3), Guerrero (2), Durango, Michoacán y Tamaulipas con una transición respectivamente. Los dos movimientos en la tasa de mujeres están en Chihuahua y Durango. La distribución de la reducción jerárquica de la tasa general se concentra en Oaxaca (17), Chihuahua (8), Michoacán (4), Durango (2) y Guerrero (2). La tasa masculina se ubica en Oaxaca (19), Chihuahua (8), Durango (3), Michoacán (3) y Guerrero (2). La disminución en la tasa femenina está en Guerrero (3) y en Chihuahua (2). Finalmente vale la pena anotar que la mayoría de las transiciones que se dan en Oaxaca tuvieron lugar entre 1990 y 2007 con una mayor frecuencia en las reducciones. Por el contrario, la mayoría de los incrementos se concentran en los estados del norte a partir de 2004. Estas diferencias en la distribución geográfica corresponden con los cambios señalados por otros trabajos sobre la distribución del homicidio en México. ${ }^{41}$

\section{Discusión}

De acuerdo con los resultados, la única forma de difusión contagiosa que se encontró con los datos disponibles es el incremento contagioso. Esta difusión, además, sigue una dirección específica: desde las áreas circundantes (o vecinas) hacia el área central o núcleo. Este tipo de transición es comparativamente más frecuente en la tasa masculina que en la femenina. En lo que concierne a los efectos transversales, los efectos con mayor prevalencia son la influencia de la tasa masculina sobre la femenina. El efecto contrario es poco frecuente y con menos peso estadístico. El otro tipo de

${ }^{41}$ F. Escalante Gonzalbo, El homicidio en México entre 1990 y 2007 aproximación estadística, México, con la colaboración de Érick Aranda, El Colegio de México, Centro de Estudios Internacionales Secretaría de Seguridad Pública Federal, 2009. 
transición detectada fue la disminución jerárquica, es decir, la disminución que ocurre entre áreas no conectadas entre sí. Los patrones detectados suceden en un número limitado de municipios; de todas las transiciones significativas hay 21 (38\%) en la tasa general, $29(44 \%)$ para la tasa masculina, $2(25 \%)$ en la femenina, $3(8 \%)$ en la transición hombre/mujer y 21 (95\%) en mujer/hombre. ${ }^{42}$

Las transiciones identificadas y su frecuencia permiten ver que la difusión contagiosa es un fenómeno real pero concentrado en un número pequeño de municipios. A pesar de ser un fenómeno característico de la tasa masculina también es un patrón relevante en la difusión de la tasa femenina. Los resultados de los efectos transversales son asimismo interesantes; la identificación de una mayor prevalencia de la difusión contagiosa en la tasa masculina que influye en la femenina concuerda con una de las características que Valdivia detectó en su análisis espacial de las diferencias entre los homicidios de hombres y mujeres. ${ }^{43}$ Los efectos de la violencia masculina en las mujeres es una relación que ya ha sido identificada en diversos trabajos. ${ }^{44}$ Por otro lado, aunque con menor frecuencia, el efecto trasversal contrario (mujeres/hombres) está presente. A pesar de que la tasa de difusión de efectos transversales es mayor en hombres/mujeres que en mujeres/hombres (porque el denominador incluye los cambios no significativos), cuando sólo se consideran las transiciones significativas y se excluye a las estacionarias, puede observase que el efecto mujeres/hombres se da en prácticamente todas las transiciones significativas de este tipo. Este es un resultado que hasta la fecha no se había mencionado en la literatura especializada y que valdría la pena estudiar con más atención.

42 Porcentajes en relación al total de transiciones significativas de todos los tipos, sin tomar en cuenta las transiciones estacionarias.

${ }^{43}$ M. Valdivia, "Análisis espacial de la dinámica del homicidio de mujeres en México a nivel municipal...”, p. 19.

${ }^{44}$ Para conocer las distintas perspectivas sobre el tema, recomiendo, para la perspectiva psicológica: Flannery, Vazsonyi y Waldman (eds.), The Cambridge Handbook of Violent Behavior, Cambridge, Cambridge University Press, 2007; y para la sociológica/criminológica: Gartner, The Oxford Handbook of Gender, Sex, and Crime, 2014. 
Otro punto a notar es que la difusión contagiosa sólo se encuentra en los incrementos y no en la disminución. Salvo lo que estudios posteriores puedan decir, el hecho de que el contagio incremental sea un patrón regular y que la disminución sea jerárquica puede tener implicaciones interesantes para la política pública de prevención y de seguridad. Por ejemplo, en lo que concierne a la política de seguridad, dado que los patrones de incremento tienen una dinámica de contagio donde el núcleo es el resultado del incremento en las áreas vecinas, tendría más sentido tomar en cuenta esta direccionalidad para diseñar estrategias de contención y evitar que se incrementen las tasas hacia las zonas colindantes. Por el contrario, si la disminución se presenta con más frecuencia de manera jerárquica, es un buen indicio para pensar en políticas de prevención destinadas a zonas con altas tasas y que no estén rodeadas de municipios con tasas altas para ayudar a la disminución de la violencia y prevenir la transición de tipo contagiosa.

Antes de hablar sobre las implicaciones que los resultados pueden tener en futuros estudios, vale la pena reconocer un límite asociado con la técnica de análisis empleada. Esta, a pesar de tener ventajas para la detección de patrones, también tiene algunas limitaciones que vale la pena tomar en cuenta. La técnica para la identificación y clasificación de transiciones entre unidades espaciales a lo largo del tiempo propuesta por Cohen y Tita es un método que se vale de las ventajas del ESDA para realizar una clasificación que no se puede lograr tan fácilmente empleando técnicas de modelación espacial. Sin embargo, también hereda sus debilidades. Una de las más importantes es el empatar la delimitación espacial con una delimitación en donde los procesos o mecanismos sociales se llevan a cabo. En este caso, el municipio como unidad de análisis no es la solución óptima porque su tamaño es muy variable y las características económicas, sociales, políticas, geográficas y de infraestructura son muy diversas entre las áreas y en su interior. Por ello es difícil justificar que los limites formales o administrativos también representan las fronteras donde se lleva acabo la interacción, los procesos y los mecanismos sociales. ¿Cuál es la mejor forma de delimitar un área para poder captar los mecanismos y procesos que hacen posible un tipo de difusión? Esta pregunta no 
es fácil de responder, pero es una asunto al que se le debe dar la atención necesaria. Por esta razón, análisis futuros que busquen trabajar sobre los procesos y mecanismos que hacen posible los patrones de difusión contagiosa que aquí se encontraron tendrán que tomar en cuenta estos límites y utilizar aproximaciones que consideren este importante detalle.

En cuanto a algunas implicaciones de los resultados para futuros estudios, el siguiente paso que se tendría que dar es hacia la identificación de los mecanismos locales asociados con las difusiones contagiosas y la manera en que estos son afectados por otro tipo de factores. Para ello hace falta examinar con mucha atención los elementos de la organización social de los incidentes criminales para entender cuáles son los procesos o mecanismos que hacen posible un tipo específico de difusión. Es en este punto donde se tienen que plantear preguntas sobre el papel de los mercados ilegales, factores institucionales como procuración de justicia y trabajo policial, factores socioeconómicos como desigualdad, empleo, educación, edad; así como disponibilidad de armas y la presencia de bandas juveniles.

Para el caso que nos ocupa, la violencia criminal y el homicidio en particular pueden estar asociados con una diferenciación mucho más vasta lo que se suele aceptar en el discurso público y académico. Por ello, es deseable que posteriores estudios hagan uso de métodos de modelación espacial que, a partir de la clasificación de transiciones que aquí se desarrolló, puedan someter a procedimientos de modelación los casos identificados incorporando a variables que la discusión suele acreditar como elementos de peso en los probables mecanismos y procesos que hacen posible la presencia de dos tipos distintos de difusiones en el territorio nacional: la difusión contagiosa y la difusión jerárquica.

Es de particular importancia seguir con esta línea de análisis ya que si la espacialidad del fenómeno de la violencia puede variar en función de variables contextuales y variables dependientes, es de esperarse que los patrones de difusión también puedan cambiar. Por ejemplo, ¿es distinta la espacialidad de la violencia cuando se controla por la presencia de bandas juveniles? ¿Pueden variar los patrones de la difusión de la criminalidad cuando se controla por 
tipo de homicidio? Hay buenos indicios en la bibliografía reciente para incorporar estas preguntas en posteriores investigaciones. Un caso interesante es el trabajo de Osorio, en donde a partir de los resultados de un coeficiente de rezago espacial (lag) se identifica que la presencia de 51 eventos de violencia semanales está asociada con un evento adicional de violencia relacionada con el tráfico de drogas en una locación determinada. Esto quiere decir que la violencia en las áreas circundantes tiene efectos positivos y significantes; la violencia no sólo está influenciada por las características locales sino también por la áreas vecinas. ${ }^{45}$ Otro buen ejemplo está en el trabajo de Dell,${ }^{46}$ donde factores contextuales como la red de caminos en una zona pueden hacer variar la dispersión de la violencia relacionada con el tráfico de drogas.

Finalmente, baste decir que este estudio buscó incorporar nuevos elementos relevantes a la investigación sobre la difusión de la criminalidad, y plantear líneas de investigación cuyo desarrollo sería de relevancia para el trabajo académico y para la orientación de políticas públicas. Esperemos que los resultados presentados alimenten el interés por estudiar las múltiples facetas de la criminalidad violenta en México.

\section{BibLIOGRAFÍA}

Abramsky, T., C. H. Watts, C. Garcia-Moreno, K. Devries, L. Kiss, M. Ellsberg, L. Heise, "What Factors Are Associated with Recent Intimate Partner Violence? Findings from the WHO Multi-Country Study on Women's Health and Domestic Violence”, BMC Public Health, vol. 11, núm. 1, 2011.

Anselin, L., Spatial Econometrics: Methods and Models, Dordrecht, Kluwer AP, 1988, en https://books.google.de/books?id=3dPIXClv4YYC

Anselin, L., "Local Indicators of Spatial Association — LISA", Geographical Analysis, vol. 27, núm. 2, 1995, pp. 93-115.

45 Osorio, "The Contagion of Drug Violence: Spatiotemporal Dynamics of the Mexican War on Drugs", Journal of Conflict Resolution, 2015, p. 14.

${ }^{46}$ Dell, op. cit. 
Anselin, L., Exploring Spatial Data with GeoDaTM: A Workbook, Urbana, University of Illinois, 2005.

Anselin, L., I. Syabri y Y. Kho, "GeoDa: An Introduction to Spatial Data Analysis”, Geographical Analysis, vol. 38, núm. 1, 2006, pp. 5-22.

Assunção, R. M. y E. A. Reis, “A New Proposal to Adjust Moran’s I for Population Density", Statistics in Medicine, vol. 18, núm. 16, 1999, pp. 21472162, en doi:10.1002/(SICI) 1097-0258(19990830) 18:16<2147::AID-SI M179>3.0.CO;2-I

Baller, R., L. Anselin, S. Messner y G. Deane, "Structural Covariates of US County Homicide Rates: Incorporating Spatial Effects", Criminology, vol. 39, 2001, p561-590, en doi:10.1111/j.1745-9125.2001.tb00 933.x

Berman, S. L., W. M. Kurtines, W. K. Silverman y L. T. Serafini, "The Impact of Exposure to Crime and Violence on Urban Youth", The American Journal of Orthopsychiatry, vol. 66, núm. 3, 1996, pp. 329-336, en http://www.ncbi.nlm.nih.gov/pubmed/8827256

Calderón, G., A. Díaz-Cayeros, B. Magaloni, G. Robles y J. Olarte, The Temporal and Spatial Dynamics of Violence in Mexico, 2012, en https:// www.dropbox.com/s/ce0lhfs9unsuqa3/paper_policies_violence_ Aug30.pdf

Castillo, J. C., D. Mejía y Restrepo, Scarcity without Leviathan: The Violent Effects of Cocaine Supply Shortages in the Mexican Drug War. Centre for Global Development - Working Papers, 2014, en http://www.cgdev.org/ sites/default/files/scarcity-leviathan-effects-cocaine-supply-shortages_1.pdf

Cliff, A. D. y J. K. Ord, "Spatial and Temporal Analysis: Autocorrelation in Space and Time”, Quantitative Geography: A British View, 1981, pp. 104-110, en http://www.scopus.com/record/display.url?eid=2-s2.00019679930 \&origin=inward\&txGid=AHR9Y36kFvzWOOZj4MYgFL $\mathrm{L} \% 3 \mathrm{a} 7$

Cohen, J. y G. Tita, "Diffusion in Homicide: Exploring a General Method for Detecting Spatial Diffusion Processes", Journal of Quantitative Criminology, vol. 15, núm. 4, 1999, pp. 451-493, en doi:10.1023/A:1007596 225550

Cummings, E. M., C. E. Merrilees, A. C. Schermerhorn, M. C. GoekeMorey, P. Shirlow y E. Cairns, "Longitudinal Pathways between Political Violence and Child Adjustment: The Role of Emotional Security 
about the Community in Northern Ireland", Journal of Abnormal Child Psychology, vol. 39, núm. 2, 2011, pp. 213-224, en doi:10.1007/s10802010-9457-3

Cummings, E. M., A. C. Schermerhorn, C. E. Merrilees, M. C. GoekeMorey, P. Shirlow y E. Cairns, "Political Violence and Child Adjustment in Northern Ireland: Testing Pathways in a Social-Ecological Model Including Single-and Two-Parent Families", Developmental Psychology, vol. 46, núm. 4, 2010, pp. 827-841, en doi:10.1037/a00 19668

Dell, M., Trafficking Networks and the Mexican Drug War, 2014, en http:// scholar.harvard.edu/dell/publications/trafficking-networks-andmexican-drug-war-0

Devries, K., C. Watts, M. Yoshihama, L. Kiss, L. B. Schraiber, N. Deyessa, C. Garcia-Moreno, "Violence against Women Is Strongly Associated with Suicide Attempts: Evidence from the WHO Multi-Country Study on Women's Health and Domestic Violence against Women", Social Science E̊ Medicine (1982), vol. 73, núm. 1, 2011, pp. 79-86, en doi:10.1016/j.socscimed.2011.05.006

Dube, A., O. Dube y O. García-Ponce, "Cross-Border Spillover: U.S. Gun Laws and Violence in Mexico", American Political Science Review, vol. 107, núm. 3, 2013, pp. 397-417, en href="http://dx.doi.org/10.1017/ S0003055413000178

Dubow, E. F., P. Boxer, L. R. Huesmann, K. Shikaki, S. Landau, S. D. Gvirsman y J. Ginges, "Exposure to Conflict and Violence across Contexts: Relations to Adjustment among Palestinian Children", Journal of Clinical Child and Adolescent Psychology, vol. 39, núm. 1, 2010, pp. 103-116, en doi:10.1080/15374410903401153

Duran-Martinez, A., Criminals, Cops, and Politicians: Dynamics of Drug Violence in Colombia and Mexico, Providence, Brown University, 2013.

Echarri-Cánovas, C. J., "Homicidio", en C. J. Echarri-Cánovas (ed.), Panorama estadístico de la violencia en México, México, El Colegio de México, 2012, pp. 51-103.

Ehrensaft, M. K., P. Cohen, J. Brown, E. Smailes, H. Chen y J. G. Johnson, "Intergenerational Transmission of Partner Violence: A 20-Year Prospective Study", Journal of Consulting and Clinical Psychology, vol. 71, núm. 4, 2003, pp. 741-753, en http://www.ncbi.nlm.nih.gov/pubmed/12924679 
Escalante Gonzalbo, F., El homicidio en México entre 1990 y 2007 aproximación estadística, México, con la colaboración de Érick Aranda, El Colegio de México, Centro de Estudios Internacionales Secretaría de Seguridad Pública Federal, 2009.

Fagan, J., Wilkinson, D. L. y Davies, G. "Social Contagion of Violence", en D. Flannery, A. Vazsonyi e I. Waldman (eds.), The Cambridge Handbook of Violent Behavior, Cambridge, Cambridge University Press, 2007, pp. 688-724, en http:/ / papers.ssrn.com/abstract=935104

Fischer, M. M. y A. Getis, Handbook of Applied Spatial Analysis: Software Tools, Methods and Applications, Berlin-Heidelberg, Springer, 2009, en https://books.google.de/books?id=c0EP_6eYsjAC

Flannery, D. J., A. T. Vazsonyi y I. D. Waldman, The Cambridge Handbook of Violent behavior and Aggression, Nueva York, Cambridge University Press, 2007.

Gartner, R. The Oxford Handbook of Gender, Sex, and Crime, 2014.

Guerra, N. G., L. R. Huesmann y A. Spindler, "Community Violence Exposure, Social Cognition, and Aggression among Urban Elementary School Children”, Child Development, vol. 74, núm. 5, 2003, pp. 15611576, en http://www.ncbi.nlm.nih.gov/pubmed/14552414

Hanson, R. F., S. Self-Brown, A. Fricker-Elhai, D. G. Kilpatrick, B. E. Saunders y H. Resnick, "Relations among Parental Substance Use, Violence Exposure and Mental Health: The National Survey of Adolescents", Addictive Behaviors, vol. 31, núm. 11, 2006, pp. 1988-2001, en http:// dx.doi.org/10.1016/j.addbeh.2006.01.012

Ingram, M. C., The Local Educational and Regional Economic Foundations of Violence: A Subnational, Spatial Analysis of Homicide Rates across Mexico's Municipalities, 2014, en http://www.wilsoncenter.org/publication/ educational-regional-foundations-violence

Ludwig, Jens y Jeffrey R. Kling, "Is Crime Contagious?”, Journal of Law and Economics, vol. 50, núm. 3, pp. 491-518, 2007, en doi:10.1086/519807

Kokko, K., L. Pulkkinen, L. R. Huesmann, E. F. Dubow y P. Boxer, "Intensity of Aggression in Childhood as a Predictor of Different Forms of Adult Aggression: A Two-Country (Finland and United States) Analysis", Journal of Research on Adolescence: The Official Journal of the Society for Research on Adolescence, vol. 19, núm. 1, 2009, pp. 9-34, en doi:10.111 1/j.1532-7795.2009.00579.x 
Krug, E. G. et al. (eds.), World Report on Violence and Health, Ginebra, World Health Organization, 2002.

LaFree, G., L. Dugan, M. Xie y P. Singh, "Spatial and Temporal Patterns of Terrorist Attacks by ETA 1970 to 2007”, Journal of Quantitative Criminology, vol. 28, núm. 1, 2011, pp. 7-29, en doi:10.1007/s10940-0119133-y

Messner, S., L. Anselin, R. Baller, D. Hawkins, G. Deane y S. Tolnay, "The Spatial Patterning of County Homicide Rates: An Application of Exploratory Spatial Data Analysis”, Journal of Quantitative Criminology, vol. 15, núm. 4, 1999, pp. 423-450, en doi:10.1023/A:100754 4208712

Morenoff, J. D., y R. J. Sampson, "Violent Crime and the Spatial Dynamics of Neighborhood Transition: Chicago, 1970-1990”, Social Forces, vol. 76, núm. 1, 1997, pp. 31-64, en doi:10.2307/2580317

National Research Council, Contagion of Violence: Workshop Summary, Washington, D. C., The National Academies Press, 2013, en http://www. nap.edu/catalog.php?record_id=13489

Osorio, J., Hobbes on Drugs: Understanding Drug Violence in Mexico, Notre Dame, University of Notre Dame, 2013.

Osorio, J., "The Contagion of Drug Violence: Spatiotemporal Dynamics of the Mexican War on Drugs", Journal of Conflict Resolution, 2015, en doi: $10.1177 / 0022002715587048$

Petras, H., K. Masyn, A. Piquero y D. Weisburd, Handbook of Quantitative Criminology, Nueva York, Springer-Verlag, 2010.

Rey, S. J., E. A. Mack y J. Koschinsky, "Exploratory Space-Time Analysis of Burglary Patterns”, Journal of Quantitative Criminology, vol. 28, núm. 3, 2011, pp. 509-531, en doi:10.1007/s10940-011-9151-9

Rios, V., How Government Structure Encourages Criminal Violence: The Causes of Mexico's Drug War, Cambridge, Harvard, 2012.

Rosenfeld, R., T. Bray y A. Egley, "Facilitating Violence: A Comparison of Gang-Motivated, Gang-Affiliated, and Nongang Youth Homicides”, Journal of Quantitative Criminology, vol. 15, núm. 4, 1999, pp. 495-516, en doi:10.1023/A:1007548309620

Smith, W. R., S. G. Frazee y E. L. Davison, "Furthering the Integration of Routine Activity and Social Disorganization Theories: Small Units of Analysis And the Study of Street Robbery As A Diffusion Process", 
Criminology, vol. 38, núm. 2, 2000, pp. 489-524, en doi:10.1111/j.17459125.2000.tb00897.x

Snyder, R. y A. Duran-Martinez, "Does Illegality Breed Violence? Drug Trafficking and State-Sponsored Protection Rackets", Crime, Law and Social Change, vol. 52, núm. 3, 2009, pp. 253-273, en doi:10.1007/ s10611-009-9195-z

Tita, G. y J. Cohen, "Measuring Spatial Diffusion of Shots Fired Activity Across City Neighborhoods”, Spatially Integrated Social Science, 2004, pp. 171-204.

Tolnay, S. E., G. Deane y E. M. Beck, "Vicarious Violence: Spatial Effects on Southern Lynchings, 1890-1919”, American Journal of Sociology, vol. 102, núm. 3, 1996, pp. 788-815, en doi:10.2307/2782463

Valdivia, M., "Análisis espacial de la dinámica del homicidio de mujeres en México a nivel municipal. Identificación y explicación de patrones de convergencia y polarización territorial", en F. Riquer y R. Castro (eds.), Estudio nacional sobre las fuentes, orígenes y factores que producen y reproducen la violencia contra las mujeres, México, Segob/Conavim, 2012.

Valdivia, M. y R. Castro, "Gender Bias in the Convergence Dynamics of the Regional Homicide Rates in Mexico", Applied Geography, vol. 45, núm. 0, 2013, pp. 280-291, en doi:http://dx.doi.org/10.1016/j.apgeog.201 3.09.015

Vilalta, C. "Un modelo descriptivo de la geografía del robo en la zona metropolitana del Valle de México", Journal of Latin American Geography, vol. 8, núm. 1, 2009, pp. 55-78.

Vilalta, C. J. "How Exactly Does Place Matter in Crime Analysis? Place, Space, and Spatial Heterogeneity", Journal of Criminal Justice Education, vol. 24, núm. 3, 2012, pp. 290-315, en doi:10.1080/10511253.20 12.715659

Vilalta, C. J., "Anomia institucional, espacialidad y temporalidad en las muertes asociadas a la lucha contra la delincuencia organizada en México", Mexican Studies/Estudios Mexicanos, vol. 29, núm. 1, 2013, pp. 280-319, en doi:10.1525/msem.2013.29.1.280

Vilalta, C. y R. Muggah, "Violent Disorder in Ciudad Juarez: A Spatial Analysis of Homicide", Trends in Organized Crime, vol. 17, núm. 3, 2014, pp. 161-180, en doi:10.1007/s12117-014-9213-0 
WHO, Global Status Report on Violence Prevention 2014, World Health Organization, the United Nations Development Programme, and the United Nations Office on Drugs and Crime, 2014.

Ye, X. y L. Wu, "Analyzing the Dynamics of Homicide Patterns in Chicago: ESDA and Spatial Panel Approaches", Applied Geography, vol. 31, núm. 2, 2011, pp. 800-807, en doi:10.1016/j.apgeog.2010.08.006 